\title{
A polo-like kinase modulates cytokinesis and flagella biogenesis in Giardia lamblia
}

\author{
Eun-Ah Park', Juri Kim ${ }^{\dagger}$, Mee Young Shin and Soon-Jung Park ${ }^{*}$
}

\begin{abstract}
Background: Polo-like kinases (PLKS) are conserved serine/threonine kinases that regulate the cell cycle. To date, the role of Giardia lamblia PLK (GIPLK) in cells has not been studied. Here, we report our investigation on the function of GIPLK to provide insight into the role of this PKL in Giardia cell division, especially during cytokinesis and flagella formation.

Methods: To assess the function of GIPLK, Giardia trophozoites were treated with the PLK-specific inhibitor GW843286X (GW). Using a putative open reading frame for the PLK identified in the Giardia genomic database, we generated a transgenic Giardia expressing hemagglutinin (HA)-tagged GIPLK and used this transgenic for immunofluorescence assays (IFAs). GIPLK expression was knocked down using an anti-g/p/k morpholino to observe its effect on the number of nuclei number and length of flagella. Giardia cells ectopically expressing truncated GIPLKs, kinase domain + linker (GIPLK-KDL) or polo-box domains (GIPLK-PBD) were constructed for IFAs. Mutant GIPLKs at Lys51, Thr179 and Thr183 were generated by site-directed mutagenesis and then used for the kinase assay. To elucidate the role of phosphorylated GIPLK, the phosphorylation residues were mutated and expressed in Giardia trophozoites

Results: After incubating trophozoites with $5 \mu \mathrm{M} \mathrm{GW}$, the percentage of cells with $>4$ nuclei and longer caudal and anterior flagella increased. IFAs indicated that GIPLK was localized to basal bodies and flagella and was present at mitotic spindles in dividing cells. Morpholino-mediated GIPLK knockdown resulted in the same phenotypes as those observed in GW-treated cells. In contrast to Giardia expressing GIPLK-PBD, Giardia expressing GIPLK-KDL was defective in terms of GIPLK localization to mitotic spindles and had altered localization of the basal bodies in dividing cells. Kinase assays using mutant recombinant GIPLKs indicated that mutation at Lys51 or at both Thr179 and Thr183 resulted in loss of kinase activity. Giardia expressing these mutant GIPLKs also demonstrated defects in cell growth, cytokinesis and flagella formation.
\end{abstract}

Conclusions: These data indicate that GIPLK plays a role in Giardia cell division, especially during cytokinesis, and that it is also involved in flagella formation.

Keywords: Giardia lamblia, Polo-like kinase, Cell cycle

*Correspondence: sjpark615@yuhs.ac

†Eun-Ah Park and Juri Kim contributed equally to this work

Department of Environmental Medical Biology and Institute of Tropical Medicine, Yonsei University College of Medicine, Seoul 03722, South Korea

\section{Background}

Giardia lamblia is a human pathogen that causes diarrheal outbreaks; it is present as either a cyst or a trophozoite. Trophozoites, the multiplying form found in hosts, possess a structure that seems to be bilaterally symmetrical from a side view and exhibits asymmetrical polarity in the anterior/posterior and dorsal/ventral views. These cells have two nuclei and cytoskeletal structures,

c) The Author(s) 2021. This article is licensed under a Creative Commons Attribution 4.0 International License, which permits use, sharing, adaptation, distribution and reproduction in any medium or format, as long as you give appropriate credit to the original author(s) and the source, provide a link to the Creative Commons licence, and indicate if changes were made. The images or other third party material in this article are included in the article's Creative Commons licence, unless indicated otherwise in a credit line to the material. If material is not included in the article's Creative Commons licence and your intended use is not permitted by statutory regulation or exceeds the permitted use, you will need to obtain permission directly from the copyright holder. To view a copy of this licence, visit http://creativecommons.org/licenses/by/4.0/. The Creative Commons Public Domain Dedication waiver (http://creativecommons.org/publicdomain/zero/1.0/) applies to the data made available in this article, unless otherwise stated in a credit line to the data. 
including an adhesive disc, a median body and four pairs of flagella [1].

Limited information is available on the mechanism responsible for regulating the division of Giardia trophozoites. Giardia lamblia reportedly has defective cell cycle checkpoints because the cell cycle of Giardia trophozoites can progress despite blocked DNA synthesis, doublestranded DNA breaks or defective mitotic spindles [2]. In vitro cultures of Giardia trophozoites are dominated by cells in the gap $2 /$ mitosis $(\mathrm{G} 2 / \mathrm{M})$ phase [3]. Investigations using synchronized cell cultures with chemicals or counterflow centrifugal elutriation have revealed that Giardia proteins show phase-specific expression [3-5]. Interestingly, a study using live imaging of Giardia cells indicated that cytokinesis occurs 60 -fold faster in Giardia than in mammalian cells, and that G. lamblia uses flagella-mediated membrane tension instead of myosin-dependent contractile rings to initiate daughter cell separation [6].

In mammals, cell division is a complex and well-organized process that incorporates a multitude of protein interactions and macromolecular machinery [7]. This process should be finely and dynamically controlled via the actions of interconnected signaling cascades, including aurora kinase (AK), polo-like kinase (PLK) and cyclin-dependent kinase 1 (CDK1) [8]. PLK is a key regulator in this process and has diverged into five paralogues in mammals, including PLK1-5 [9]. In particular, PLK1 is a mitotic kinase with multiple roles in several steps of the cell cycle from G2 to the final step of cytokinesis [10]. These Ser/Thr kinases are defined by the presence of an $\mathrm{N}$-terminal kinase domain (KD) and additional domains, termed polo-box domains (PBDs), which engage in protein interactions [11]. To perform its functions, PLK must be activated and dynamically recruited to distinct subcellular structures spatially and temporally via its interaction with the PBD [12].

An investigation of the Giardia kinome indicated the presence of an open reading frame (ORF) for PLK, named GL50803_104150 [13]. In the present study, we examined the putative role of PLK using a PLK inhibitor as well as morpholino-mediated knockdown with respect to G. lamblia cell division. The autophosphorylation activity of G. lamblia PLK (GlPLK) was measured in vitro, and its role in cell division was also confirmed in vivo using transgenic G. lamblia ectopically expressing a mutant GIPLK that lacks critical residue(s) for autophosphorylation.

\section{Methods}

\section{Culture of G. lamblia trophozoites}

Giardia lamblia trophozoites (strain WB, ATCC30957; American Type Culture Collection, Manassas, VA, USA) were grown in modified TYI-S-33 medium ( $2 \%$ casein digest, $1 \%$ yeast extract, $1 \%$ glucose, $0.2 \% \mathrm{NaCl}, 0.2 \%$ L-cysteine, $0.02 \%$ ascorbic acid, $0.2 \% \mathrm{~K}_{2} \mathrm{HPO}_{4}, 0.06 \%$ $\mathrm{KH}_{2} \mathrm{PO}_{4}, 10 \%$ calf serum and $0.5 \mathrm{mg} / \mathrm{mL}$ bovine bile, $\mathrm{pH}$ 7.1) at $37^{\circ} \mathrm{C}$ [14].

\section{Scoring of G. lamblia cells for cell growth}

The $50 \%$ inhibitory concentration for cell death $\left(\mathrm{IC}_{50}\right)$ was determined by treating Giardia trophozoites $\left(2 \times 10^{4}\right.$ cells $\left./ \mathrm{ml}\right)$ with various concentrations $)(5-15 \mu \mathrm{M})$ of the PLK inhibitor, GW843682X (GW; Cayman Chemical, Ann Arbor, MI, USA). After treatment for $24 \mathrm{~h}$, the number of parasites per milliliter was determined using a hemocytometer. Giardia trophozoites treated with $0.3 \%$ dimethyl sulfoxide (DMSO) were used as controls.

Various Giardia cells (trophozoites carrying plasmids pKS-3HA.neo, pGlPLK.neo, pGIPLKK51R.neo or pGIPLKT179AT183A.neo) were inoculated into modified TYI-S-33 medium at $1 \times 10^{4}$ cells $/ \mathrm{ml}$, and the cell numbers were counted every $6 \mathrm{~h}$ for up to $60 \mathrm{~h}$ using a hematocytometer.

\section{Microscopic observation of Giemsa-stained cells}

For the microscopic observation of cells, the cells were attached to slides, air-dried and fixed with $100 \%$ methanol for $10 \mathrm{~min}$, following which they were stained with $10 \%$ Giemsa solution for $40 \mathrm{~min}$ and washed with distilled water. After mounting with dibutyl phthalate xylene (Sigma-Aldrich, St. Louis, MO, USA), the slides were observed under an Axiovert 200 microscope (Carl Zeiss AG, Oberkochen, Germany). For each condition, at least 300 cells were examined to determine the number and position of the nuclei. Among cells with two nuclei in normal positions, the number of cells showing nuclei condensation was also recorded. Data are presented as the mean \pm standard deviation (SD) of three independent experiments.

To measure flagella length, we observed Giemsastained cells under an Axiovert 200 microscope and analyzed their differential interference contrast (DIC) images using Fiji, an open-source platform for biological-image analysis [15]. Each of four types of flagella were divided into cytoplasmic and membrane-bound categories and then measured using the line Freehand Tracing 0mode in ImageJ software (http://imagej.nih.gov/ij/). These data for flagella length were derived from 35 cells per each experiment. Data are presented as the mean \pm SD of three independent experiments.

\section{Flow cytometry}

Both the GW-treated and control G. lamblia cells were analyzed for their DNA content using flow cytometry [16]. Briefly, the harvested cells were resuspended in 
$50 \mu \mathrm{l}$ TYI-S-33 culture medium and treated with $150 \mu \mathrm{l}$ of a cell fixative (1\% Triton X-100, $40 \mathrm{mM}$ citric acid, $20 \mathrm{mM}$ dibasic sodium phosphate, $200 \mathrm{mM}$ sucrose; $\mathrm{pH}$ 3.0) at room temperature for $5 \mathrm{~min}$. The samples were diluted with $350 \mu \mathrm{l}$ of diluent buffer [125 mM $\mathrm{MgCl}_{2}$ in phosphate-buffered saline (PBS: $137 \mathrm{mM}$ $\mathrm{NaCl}, 2.7 \mathrm{mM} \mathrm{KCl}, 10.1 \mathrm{mM} \mathrm{Na}_{2} \mathrm{HPO}_{4}$ and $2 \mathrm{mM}$ $\mathrm{KH}_{2} \mathrm{PO}_{4}, \mathrm{pH}$ 7.4)] and then stored at $4{ }^{\circ} \mathrm{C}$ until use. Fixed cells were treated with $2.5 \mu \mathrm{g}$ RNase A (SigmaAldrich) and $10 \mu \mathrm{g} / \mathrm{ml}$ propidium iodide (PI; SigmaAldrich) for $30 \mathrm{~min}$ at $37^{\circ} \mathrm{C}$. These data obtained via flow cytometry were analyzed using FlowJo software version 10.2 (FlowJo Llc, Ashland, OR, USA). Along representative histograms, averages of three independent experiments were presented in bar graphs.
Construction of G. Iamblia expressing the hemagglutinin (HA) epitope-tagged GIPLK proteins

A 2184-bp DNA fragment of the glplk gene, which comprises the promoter region (150 bp) and the ORF, was amplified from Giardia genomic DNA by PCR using two primers, Pplk-F and PLK-PDB-R (Table 1). The NotI and Sall sites were cloned into plasmid pKS-3HA.neo [17] to obtain pGIPLK.neo. The construct was confirmed by DNA sequencing using a sequencing service company (Macrogen, Seoul, Korea).

An aliquot $(20 \mu \mathrm{g})$ of pGlPLK.neo was transfected into $1 \times 10^{7}$ Giardia trophozoites by electroporation under the following conditions: $350 \mathrm{~V}, 1000 \mu \mathrm{F}$ and $700 \Omega$ (Bio-Rad, Hercules, CA, USA). Expression of HA-tagged GlPLK was confirmed by western blotting. Giardia trophozoites carrying pKS-3HA.neo were included as empty vectors. For each experiment, five sets of transfection were performed with an $80 \%$ success rate. Three of

Table 1 Primers and morpholinos used in this study

\begin{tabular}{|c|c|}
\hline Name & Nucleotide sequence $\left(5^{\prime}-3^{\prime}\right)^{\mathrm{a}, \mathrm{b}}$ \\
\hline \multicolumn{2}{|c|}{ Transgenic Giardia lamblia expressing HA-tagged GIPLK } \\
\hline Pplk-F & CATCGCGGCCGCGTAGGCGTCATCCGAGGTGAAC \\
\hline PLK-NL-R & GTTACGTCGACGTCTATTTGTGGATACTCGGCTT \\
\hline Pplk-R & GTTACAAGCTTGGGGCTATAAAATTTTACAGAG \\
\hline PLK-PBD-F & GTTACAAGCTTCCACCGTGCTACATCATGTCCTG \\
\hline PLK-PBD-R & GTTACGTCGACTCCCCTCCCTGACCGAGCTGCCT \\
\hline \multicolumn{2}{|c|}{ Mopholino sequences } \\
\hline Control & ССTCTTACCTCAGTTACAATTTATA \\
\hline Anti-glplk & AGCTCCCACCGCAAAAGCCAAAATT \\
\hline \multicolumn{2}{|c|}{ Real-time PCR primers } \\
\hline PLK-RT-F & GTCACGTTTATGAGCGAGAA \\
\hline PLK-RT-R & CTATTCCCCTCCCTGACCGA \\
\hline Actin-F & GTCCGTCATACCATCTGTTC \\
\hline Actin-R & GTTTCCTCCATACCACACG \\
\hline \multicolumn{2}{|l|}{ Kinase assay } \\
\hline PLK-GBK-F & GCACGAATTCATGTCCCACAGCAACGCCCCAGAA \\
\hline PLK-GBK-R & CTACAGCGGCCGCCTATTCCCCTCCCTGACCGAGCT \\
\hline \multicolumn{2}{|c|}{ Mutagenesis of $g / p / k$} \\
\hline PLKT183A-F & TGGGCCATGTGTGGAGCGCCAAACTTT \\
\hline PLKT183A-R & GAGAAAGTTTGGCGCTCCACACAT \\
\hline \multicolumn{2}{|c|}{ Recombinant protein for antibodies } \\
\hline rGIPLK-F & GATCGAATTCGATGTCCCACAGCAACGCCCCA \\
\hline rGIPLK-R & CGATCTCGAGTTCCCCTCCCTGACCGAGCTG \\
\hline rGIGAP1-F & GATTGAATTCATGCCTATTCGCCTCGGAAT \\
\hline rGIGAP1-R & GCCTAGCGGCCGCGGCAGCCCTTGGACCCGACGTA \\
\hline rGICENH3-F & GATCCATATGATGAGCGGAGGCTCACGG \\
\hline rGICENH3-R & TACCGTCGACCCGTAGTGAATTTAAGTT \\
\hline
\end{tabular}

glplk Giardia lamblia polo-like kinase gene, HA Hemagglutinin

${ }^{a}$ Restriction enzyme sites are underlined

${ }^{b}$ Mutated bases are indicated as bold and italic letters 
the resulting transfectants were examined for the expression of HA-tagged GIPLK.

Two truncated GIPLK proteins were also ectopically expressed in Giardia trophozoites. A 1443-bp DNA fragment encoding the N-terminal portion of GlPLK (KD+linker region) was amplified using the primers Pplk-F and PLK-NL-R (Table 1) and then cloned into the NotI and SalI sites of pKS-3HA.neo to generate pGIPLKKDL.neo. To express the two PBDs of GlPLK, a 150-bp glplk promoter region (amplified by PCR using primers Pplk-F and Pplk-R) was cloned into pKS-3HA.neo to produce pPplk-3HA.neo. Subsequently, DNA encoding the PBDs of G1PLK was amplified using the PLK-PBD-F and PLK-PBD-R primers and then cloned into the HindIII and SalI regions of pPplk-3HA.neo to obtain pGIPLKPBD.neo. These plasmids were transfected into Giardia trophozoites as described above. The expression of these truncated proteins was examined by western blotting using anti-HA antibodies.

\section{Western blotting}

Extracts prepared from Giardia cells carrying plasmids pKS-3HA.neo or pGIPLK.neo were separated via sodium dodecyl sulfate-polyacrylamide gel electrophoresis (SDS-PAGE) and transferred onto a polyvinylidene fluoride membrane (Millipore, Bedford, MA, USA). The membrane was incubated with monoclonal mouse antiHA antibodies (1:1000; Sigma-Aldrich) in TBST solution (Tris-buffered saline with Tween 20; $50 \mathrm{mM}$ Tris- $\mathrm{HCl}$, $5 \%$ skim milk and $0.05 \%$ Tween 20 ) at $4{ }^{\circ} \mathrm{C}$ overnight. The membranes were then incubated with horseradish peroxidase-conjugated secondary antibodies, and immunoreactive proteins were visualized using an enhanced chemiluminescence system (Thermo Fisher Scientific, Waltham, MA, USA). Membranes were incubated in a stripping buffer (Thermo Fisher Scientific) at room temperature for $20 \mathrm{~min}$ and then reacted with polyclonal rat antibodies against protein disulfide isomerase 1 (PDI1; GL50803_29487) of G. lamblia (1:10,000) as the loading control [18].

\section{Immunofluorescence assay}

To obtain more dividing cells for immunofluorescence assays (IFAs), the culture medium of Giardia trophozoites grown for 3 days was replaced with fresh medium, and the cells were collected after growing for 3-4 h. Giardia cells were attached onto glass slides coated with L-lysine for $10 \mathrm{~min}$ and then fixed with chilled methanol for 10 min followed by PBS/0.5\% Triton X-100 for $10 \mathrm{~min}$. After blocking for $1 \mathrm{~h}$ in $\mathrm{PBS} / 5 \%$ goat serum $/ 3 \%$ bovine serum albumin, the cells were incubated with primary antibodies overnight at $4{ }^{\circ} \mathrm{C}$ and subsequently treated with fluorescent dye-conjugated secondary antibodies. The samples were mounted with ProLong ${ }^{\mathrm{TM}}$ Gold Antifade Mountant with DAPI (Molecular Probes, Waltham, MA, USA) and then examined inverted confocal laser scanning microscopy (model LSM700 microscrope; Carl Zeiss AG).

The following antibodies were used at the indicated dilutions: anti-HA mouse monoclonal antibodies (1:100; Sigma-Aldrich), anti-HA rat monoclonal antibodies (1:100; clone 3F10, Roche Applied Science, Mannheim, Germany), anti-acetylated- $\alpha$-tubulin mouse antibodies (1:800; T7451, clone 6-11B-1, Sigma-Aldrich), antiGlCentrin rat antibodies (1:100; [18]), Alexa Fluor 488-conjugated goat anti-mouse IgG (1:100; Molecular Probes), Alexa Fluor 488-conjugated goat anti-rat IgG (1:400; Molecular Probes), Alexa Fluor 555-conjugated anti-rat IgG (1:100; Molecular Probes) and Alexa Fluor 568-conjugated anti-mouse IgG (1:100; Molecular Probes).

Antibodies specific to the phosphorylated form of PLK were purchased from Abcam (ab39068; Cambridge, MA, USA) and then used for IFA of G. lamblia cells (1:100) along with anti-HA antibodies to discern the localization of phosphorylated GIPLK.

\section{Morpholino knockdown}

Expression of GIPLK was knocked down using a morpholino, as described previously [19]. A specific morpholino for GIPLK was designed by Gene Tools (Philomath, OR, USA) (see Table 1 for sequences). Nonspecific oligomers were used as a control morpholino (Table 1). Cells $\left(5 \times 10^{6}\right.$ in $0.3 \mathrm{ml}$ medium) were treated with the lyophilized morpholino at a final concentration of $100 \mathrm{nM}$. After electroporation, the cells were grown for various lengths of time, ranging from 12 to $48 \mathrm{~h}$, and analyzed for GlPLK inhibition by western blotting using anti-HA and anti-GlPLK antibodies. The cells at $18 \mathrm{~h}$ post-transfection were analyzed for their nuclear phenotypes, including the number and position of nuclei and condensation and flagella length, as described above. In addition, cells transfected with control or anti-glplk morpholino were harvested at 6 or $18 \mathrm{~h}$, and then evaluated with respect to their DNA content by flow cytometry followed by analysis using FlowJo software (FlowJo Llc). Transfection was performed at least five times, and the data presented were derived from three independent knockdown experiments.

\section{Cell cycle synchronization in G. lamblia using nocodazole and aphidicolin}

Giardia trophozoites $\left(5 \times 10^{5}\right.$ cells $\left./ \mathrm{ml}\right)$ were incubated in modified TYI-S-33 medium to $60 \%$ confluency. A portion of these cells was treated with $100 \mathrm{nM}$ nocodazole (Sigma-Aldrich) for $2 \mathrm{~h}$ and harvested as G2/M-phase 
cells. The remaining nocodazole-treated cells were treated with $6 \mu \mathrm{M}$ aphidicolin (Sigma-Aldrich) for $6 \mathrm{~h}$ to obtain gap 1/synthesis (G1/S)-phase cells. Giardia trophozoites treated with $0.01 \%$ DMSO instead of nocodazole and aphidicolin were used as controls.

These cells were then analyzed by flow cytometry to determine the ploidy of their DNA. Intracellular levels of GIPLK protein in the DMSO-treated, nocodazole-treated and nocodazole/aphidicolin-treated Giardia cells were determined by western blotting. The intracellular levels of $g l p l k$ transcripts were also measured in these cells.

\section{Formation of anti-GIPLK, anti-GIGAP and anti-CenH3 antibodies}

A 2037-bp glplk DNA fragment was amplified by PCR using the primers rGIPLK-F and rGIPLK-R (Table 1), and then cloned into pET32b to produce pET32-GIPLK (Table 2).

In Giardia, a conventional histone 3 and two histone 3 variants, i.e. histone $3 \mathrm{~B}$ and centromeric histone 3 , have been reported, all of which were found in the nuclei when they are expressed as green fluorescent protein-tagged proteins in vivo [20]. One of the histone 3 proteins, i.e. centromeric $\mathrm{H} 3$, was expressed as a recombinant protein and then used to make antibodies for a marker for the nuclear protein. A 1011-bp DNA fragment encoding GlGAP1 (GL50803_6687) or a 471-bp DNA fragment encoding GlCenH3 (GL50803 20037) were amplified from the Giardia genome. Each fragment was cloned into pGEX4T-1 or pET21b to produce pGEX-GIGAP1 or pET-GlCENH3, respectively (Table 2). Histidine-tagged GIPLK, GST-GlGAP1 and HA-tagged GlCenH3 were overexpressed in Escherichia coli BL21 (DE3) with the

Table 2 Strains and plasmids used in this study

\begin{tabular}{|c|c|c|}
\hline Organism/plasmid & Description & Source/references \\
\hline \multicolumn{3}{|l|}{ Giardia lamblia } \\
\hline ATCC 30957 & Clinical isolate & ATCC \\
\hline \multicolumn{3}{|l|}{ Escherichia coli } \\
\hline $\mathrm{DH} 5 \mathrm{a}$ & $\begin{array}{l}\text { supE44, } \triangle \text { lacU169 ( } \$ 80 \text { lacZ } \triangle M 15), \text { hsdR17, recA1, endA1, } \\
\text { gyrA96, thi-1, relA1 }\end{array}$ & Invitrogen, Thermo Fisher Scientific (Carlsbad, CA, USA) \\
\hline BL21 (DE3) & $F^{\prime}$, ompt, hsdSB(rB- $\left.m B^{-}\right)$gal, dcm (DE3) & Invitrogen, Thermo Fisher Scientific \\
\hline \multicolumn{3}{|l|}{ Plasmids } \\
\hline pKS-3HA.neo & Shuttle vector, $\mathrm{Amp}^{\mathrm{R}}$, neo gene & {$[17]$} \\
\hline pGIPLK.neo & $\begin{array}{l}\text { pKS_3HA.neo, } 2184 \text { bp, encoding glplk (GiardiaDB ID } \\
\text { GL50803_104150) }\end{array}$ & This study \\
\hline pGIPLKKDL.neo & $\begin{array}{l}\text { pKS-3HA.neo, } 1443 \text { bp, encoding kinase domain and linker of } \\
\text { glplk }\end{array}$ & This study \\
\hline pPplk-3HA.neo & pKS-3HA.neo, 150 bp, encoding promoter region of glplk & This study \\
\hline pGIPLKPBD.neo & $\begin{array}{l}\text { pKS-3HA.neo, } 894 \text { bp, encoding promoter region and PBDs of } \\
\text { glplk }\end{array}$ & This study \\
\hline pGIPLKK51R.neo & pKS-3HA.neo, 2184 bp, encoding K51R glplk & This study \\
\hline pGIPLKT179A.neo & pKS-3HA.neo, 2184 bp, encoding T179A glplk & This study \\
\hline pGIPLKT183A.neo & pKS-3HA.neo, 2184 bp, encoding T183A glplk & This study \\
\hline pGIPLKT179AT183A.neo & pKS-3HA.neo, 2184 bp, encoding T179AT183A glplk & This study \\
\hline pGBKT7 & Gal4p $p_{(1-147)}$ DNA-BD, TRP1, Kan ${ }^{R}$, c-Myc Epitope & Clontech, Takara Bio (Mountainview, CA, USA) \\
\hline pGBK-GIPLK & pGBKT7, 2037 bp, encoding glplk & This study \\
\hline pGBK-GIPLKK51R & pGBKT7, 2037 bp, encoding K51R glplk & This study \\
\hline pGBK-GIPLKT179A & pGBKT7, 2037 bp, encoding T179A glplk & This study \\
\hline pGBK-GIPLKT183A & pGBKT7, 2037 bp. encoding T183A glplk & This study \\
\hline pGBK-GIPLKT179AT183A & pGBKT7, 2037 bp, encoding T179AT183A glplk & This study \\
\hline pET32b & Expression vector, $\mathrm{Amp}^{\mathrm{R}}$ & $\begin{array}{l}\text { Novagen (Merck Biosciences, Merck AG (Darmstadt, } \\
\text { Germany) }\end{array}$ \\
\hline pET32-GIPLK & pET32b, 2037 bp, encoding GIPLK & This study \\
\hline pGEX4T-1 & Expression vector, Amp ${ }^{R}$, GST & GE Healthcare (Chicago, IL, USA) \\
\hline pGEX-GIGAP1 & pGEX4T-1, 1011 bp, encoding glgap 1 & This study \\
\hline pET21b & Expression vector, $\mathrm{Amp}^{\mathrm{R}}$ & Novagen \\
\hline pET-GICENH3 & pET21b, 471 bp, encoding glcenh3 & This study \\
\hline
\end{tabular}


addition of $1 \mathrm{mM} \mathrm{IPTG}$ at $37^{\circ} \mathrm{C}$. The resultant recombinant proteins were excised from the SDS-PAGE gel and used to immunize Sprague-Dawley rats (2 weeks old, female) to produce polyclonal antibodies, as previously described [21]. All primers used are listed in Table 1.

\section{Quantitative real-time PCR}

Total RNA was prepared from interphase, G1/S-phase and G2/M-phase cells using TRIzol (Invitrogen, Thermo Fisher Scientific, Carlsbad, CA, USA) according to the manufacturer's instructions. A 5- $\mu$ g aliquot of RNA was converted into complementary DNA (cDNA) using an Improm-II Reverse Transcription System (Promega, Madison, WI, USA). Real-time PCR was performed using a LightCycler System and LightCycler 480 SYBR Green I Master Kit (Roche Applied Science, Penzberg, Germany). The conditions for real-time PCR were as follows: preincubation at $95{ }^{\circ} \mathrm{C}, 5 \mathrm{~min}$; then $94{ }^{\circ} \mathrm{C} / 1 \mathrm{~min}, 56^{\circ} \mathrm{C} / 1 \mathrm{~min}$, $72{ }^{\circ} \mathrm{C} / 1 \mathrm{~min}$ for 45 cycles. The nucleotide sequences of the forward and reverse primers used for real-time PCR are listed in Table 1. The G. lamblia actin-related gene (glactin; GL50803_15113) transcript was used to normalize the amount of mRNA in the samples, which has been reported to be constitutively expressed during the cell cycle of G. lamblia [5]. All experiments were performed with three independently prepared cultures.

\section{Subcellular protein fractionation}

Giardia lamblia cells at various cell cycle phases (interphase, G1/S-phase and G2/M-phase cells; $2 \times 10^{9}$ cells) were lysed in hypotonic buffer [10 mM HEPES-KOH, $10 \mathrm{mM} \mathrm{KCl}, 1.5 \mathrm{mM} \mathrm{MgCl}, 0.2 \mathrm{mM}$ PEFA1023 (pH 7.9), $0.5 \%$ Nonidet P-40 (NP-40), $20 \mathrm{mM}$ NEM, protease inhibitor cocktail], as described previously [22]. After centrifugation for $10 \mathrm{~min}$ at $16,000 \mathrm{~g}$, supernatants were collected as cytoplasmic extracts. The pellets were treated with high-salt buffer $(450 \mathrm{mM} \mathrm{NaCl}, 50 \mathrm{mM}$ Tris-HCl, 2 mM DTT, 1\% NP-40, 20 mM NEM, protease inhibitor cocktail) for $10 \mathrm{~min}$ and then centrifuged at $4{ }^{\circ} \mathrm{C}$ for $15 \mathrm{~min}$ at 16,000 $\mathrm{g}$. The supernatants were collected as membrane extracts. Equal amounts of cytoplasmic and membrane extracts were analyzed by western blotting using anti-HA (1:1000), anti-GlGAP1 (Gl50803_6687; 1:10,000), anti-GlCentrin (Gl50803_104685; 1:10,000; [18]) or anti-GlCenH3 antibodies (GL50803_20037; 1:5000).

\section{In vitro transcription/translation synthesis of rGIPLK proteins}

The TNT T7 Coupled Reticulocyte Lysate System (Promega) was used for the in vitro synthesis of c-Myc-tagged GIPLK. The DNA template $(0.5 \mu \mathrm{g}$; pGBK-GIPLK, pGBKGIPLKK51R, pGBK-GIPLKT179A, pGBK-GIPLKT183A and pGBK-GIPLKT179AT183A) was incubated with the transcription/translation mix in a total volume of $50 \mu \mathrm{l}$ at $30{ }^{\circ} \mathrm{C}$ for $90 \mathrm{~min}$. The synthesized protein products were resolved by SDS-PAGE and analyzed by western blotting with mouse anti-c-My antibodies (1:1000; Santa Cruz Biotechnology, Dallas, TX, USA).

\section{Kinase assay}

The rGlPLK proteins, which were prepared as mentioned above, were resuspended in $20 \mu \mathrm{l}$ kinase buffer $(50 \mathrm{mM}$ Tris- $\mathrm{HCl}, 10 \%$ glycerol, $5 \mathrm{mM} \mathrm{MgCl}, 150 \mathrm{mM} \mathrm{NaCl}$, $50 \mathrm{mM} \mathrm{KCl}$ and $1 \mathrm{mM}$ DTT, $\mathrm{pH} 8.0$ ) and then used for kinase assays in the presence of $2.5 \mu \mathrm{Ci}\left[\gamma^{-32} \mathrm{P}\right] \mathrm{ATP}$ (3000 Ci/mmol; PerkinElmer, Waltham, MA, USA). The kinase reactions were processed for $30 \mathrm{~min}$ at $30^{\circ} \mathrm{C}$ and then stopped by adding SDS loading buffer. Samples were separated in 12\% SDS-PAGE gels, which were then dried and subjected to autoradiography.

\section{Generation of mutant GIPLK proteins by site-directed mutagenesis}

As candidate sites for phosphorylation, Lys51 was modified to Arg, whereas Thr179 and Thr183 were mutated to Ala. The following plasmids were supplied by Macrogen for the in vitro synthesis of rGlPLK and the in vivo expression of GlPLK in Giardia: pGBK-GIPLKK51R, pGBK-GIPLKT179A, pGBK-GIPLKT183A (for in vitro synthesis), pGIPLK51R.neo, pGIPLKT179A.neo and pGIPLKT184A.neo (for expression in Giardia). Plasmids for mutant rGIPLKT179AT183A were generated by site-directed mutagenesis using primers carrying the substitution. To generate a plasmid for the expression of the T179AT183A double mutant GlPLK in Giardia, two DNA fragments were amplified using the pGIPLKT179A. neo plasmid as a template with two primer sets, Pplk-F/ PLKT183A-R or PLKT183A-F/PLK-PBD-R. The resulting PCR products were used as templates for a second round of PCR with the primers Pplk-F and PLK-PBD-R. The DNA fragment was then cloned into pKS-3HA.neo, resulting in the pGIPLKT179AT183A.neo plasmid. The plasmid for in vitro synthesis of the T179AT183A double mutant rGlPLK was constructed in the same manner. Briefly, two PCR fragments were amplified using PLK-GBK-F/PLKT183A-R or PLKT183A-F/PLK-GBK-R. Using these DNA fragments as templates, a second round of PCR was performed with the primers PLK-GBK-F and PLK-GBK-R to obtain the pGBK-PLKT179AT183A plasmid.

These constructed plasmids for the in vivo expression of mutant GlPLKs in Giardia were transfected into trophozoites by electroporation and the resulting strains were examined for their nuclear phenotypes and flagella length. 


\section{Statistical analysis}

Data are presented as the mean \pm SD of three independent experiments. Statistical analyses for pairwise comparisons were performed using Student's t-test to evaluate the statistical significance of these results. Differences with $P$ values of $<0.05$ were considered to be significant. Data with $P$ values of $<0.01$ are indicated with two asterisks, whereas data with $P$ values between 0.01 and 0.05 are indicated with a single asterisk.

\section{Results}

Inhibition of PLK activity affects the cell cycle and flagella biogenesis in G. lamblia

In order to define the role of PLK, we treated G. lamblia trophozoites with various concentrations of GW843682X $(\mathrm{GW})$, an ATP-competitive inhibitor of PLK1 and PLK3 (Additional file 1: Fig. S1) and observed that the inhibition of growth of G. lamblia was inversely related to the GW concentration, with an $\mathrm{IC}_{50}$ of $7 \mu \mathrm{M}$.

To determine the effect of PLK inhibition on Giardia cell division, the cells were treated with $5 \mu \mathrm{M} \mathrm{GW}$ for various lengths of time (range 3-24 h), then stained with Giemsa. The stained cells ( $>300$ cells for each of the three independent experiments) were scored for the number and position of the nuclei, as follows: cells with one nucleus, with two nuclei in the normal position, with two nuclei in an abnormal position, with three nuclei, with four nuclei and with $>4$ nuclei (Additional file 2: Table S1). Based on these data, treatment with $5 \mu \mathrm{M}$ $\mathrm{GW}$ for $18 \mathrm{~h}$ was selected to determine the number of nuclei in GW-treated cells and control cells (treated with DMSO for $18 \mathrm{~h}$ ) (Fig. 1a). A representative cell for each category is shown in Fig. 1a. A majority of the cells were found to have two nuclei in the normal position (97\%), and the percentage of these cells decreased to

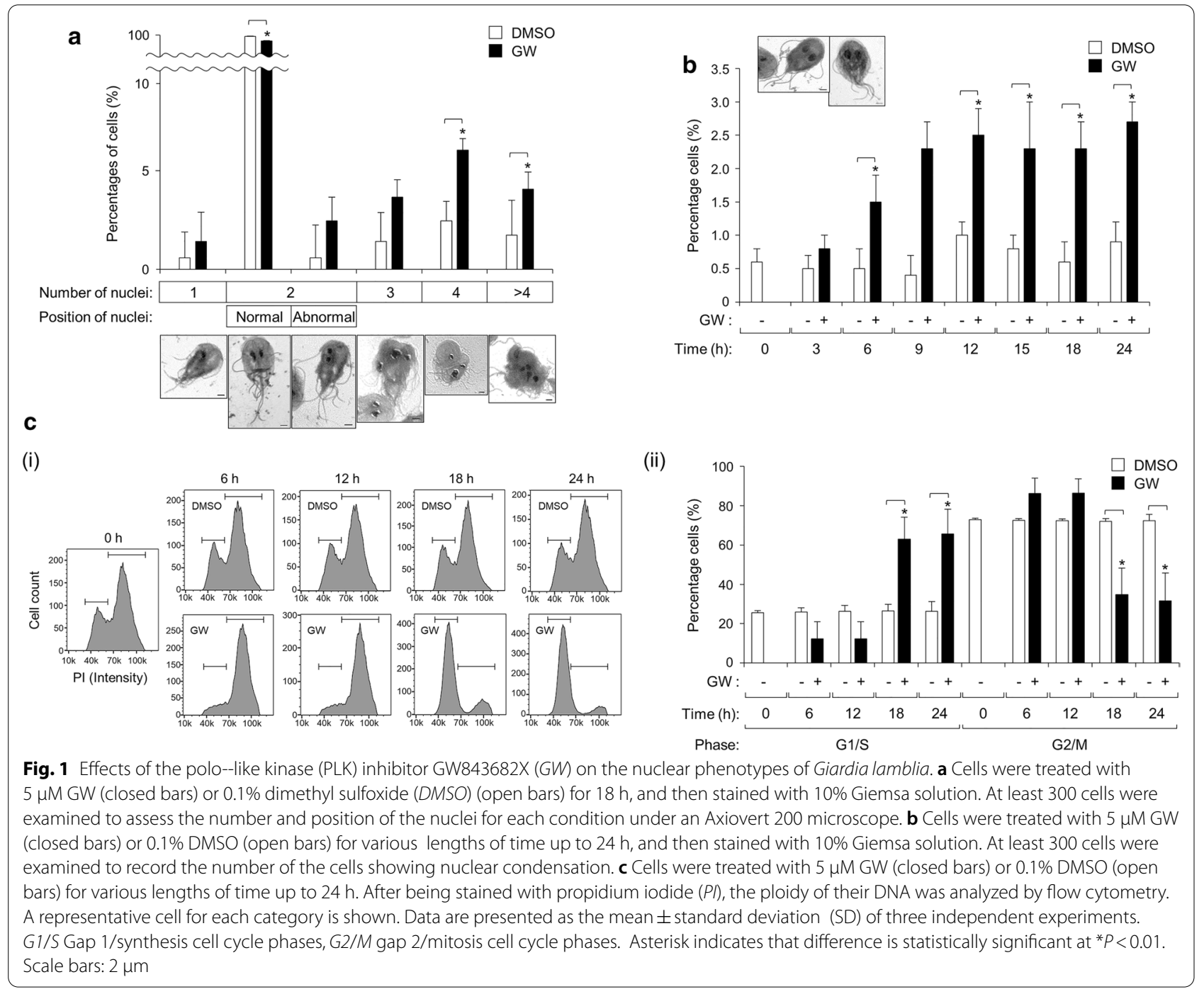


$87 \%$. The number of cells with one nucleus, two abnormally positioned nuclei or three nuclei increased, but this change was not statistically significant. The most notable increase was the percentage of cells with $\geq 4$ nuclei, in particular to 5.8 and $2.2 \%$ (from 1 and $0.7 \%$ of the control cells, respectively), indicating that $\mathrm{GW}$ induced cell cycle arrest during cytokinesis.

Among the GW-treated Giardia trophozoites with two nuclei in the normal position, we found cells with condensed nuclei that which appeared larger and more deeply stained. Thus, the percentages of cells with condensed nuclei were also monitored in the cells treated with $5 \mu \mathrm{M} \mathrm{GW}$ for various lengths of time $(3-24 \mathrm{~h})$ (Fig. 1b). The percentage of cells with condensed nuclei significantly increased in cells treated with $5 \mu \mathrm{M} \mathrm{GW}$ for $6 \mathrm{~h}$ compared to DMSO-treated cells.

In order to determine the effect of GIPLK inhibition on DNA content of Giardia, we treated trophozoites with $0.1 \%$ DMSO or $5 \mu \mathrm{M} \mathrm{GW}$ for various lengths of time $(6,12,18$ and $24 \mathrm{~h})$ and then analyzed the tropozoites by flow cytometry (Fig. 1c). The presented data are representatives of three independent samples per each condition. Control cells (untreated cells and DMSOtreated cells) were found to be a mixture of G1/S-, and G2/M-phase cells, with the cells at the G2/M phase the most dominant (72-73\%), as reported previously [3]. For cells treated with GW for 6 and $12 \mathrm{~h}$, the percentage of cells at G2/M phase increased to $86 \%$, which was not a statistically significant difference. Interestingly, a greater number of cells treated with $5 \mu \mathrm{M} \mathrm{GW}$ for longer times, 18 and $24 \mathrm{~h}$, were present in the G1/S phase (63-66\%), as compared with the control cells (26\%). These results indicated that the inhibition of PLK in Giardia causes cell cycle arrest at the G2/M phase or the G1/S phase, depending upon the treatment time with GW.

In addition, we examined whether the GW treatment affected the flagella formation of Giardia trophozoites (35 cells per each condition, and 3 independent experiments) by quantitatively measuring the length of the membrane-bound and cytoplasmic portion of all four pairs of flagella (Fig. 2a). The cytoplasmic portion of the ventral flagella could not be measured in this assay. The length of the cytoplasmic posterolateral flagella was 5.2$7.2 \mu \mathrm{m}$. The anterior and caudal flagella had longer cytoplasmic portions (7.9-9.2 and 7.9-10.8 $\mu \mathrm{m}$, respectively). None of the three flagella showed any significant change in the length of their cytoplasmic part in response to GW.

With respect to the membrane-bound region, the lengths of the four flagella were more variable. Under normal conditions, that is, DMSO treatment for various time periods, the membrane-bound ventral flagella were the longest $(13.8 \mu \mathrm{m})$. The caudal flagella demonstrated the shortest membrane-bound region $(7.2 \mu \mathrm{m})$. The lengths of the posterolateral and anterior membrane flagella were 7.9 and $9.0 \mu \mathrm{m}$, respectively. The effect of GW on the length of the membrane-bound flagella was determined using data derived from the cells treated with GW for $18 \mathrm{~h}$ and their counterpart control cells (Fig. 2b).
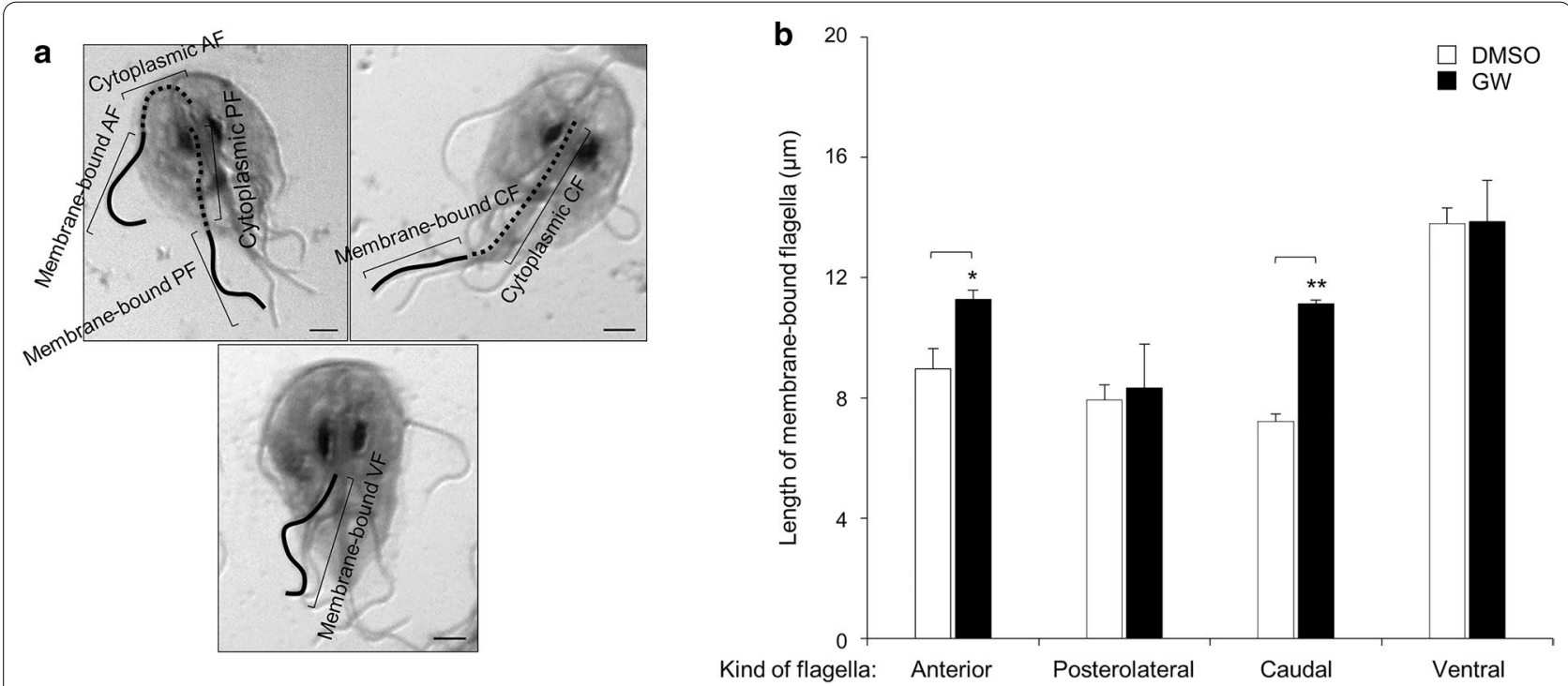

Fig. 2 Effects of the PLK inhibitor GW on flagella formation in G. lamblia. Cells were treated with 5 MM GW (closed bars) or $0.1 \%$ DMSO (open bars) for $18 \mathrm{~h}$, and then stained with Giemsa solution. a Representative figures showing how to measure the cytoplasmic anterior flagella (AF), membrane-bound AF, cytoplasmic posterolateral flagella (PF), membrane-bound PF, cytoplasmic caudal flagella (CF), membrane-bound CF and membrane-bound ventral F (VF). Scale bars: $2 \mu \mathrm{m}$. b Flagellar length was measured in 35 cells per each condition. Data are presented as the mean of three independent experiments. Asterisks indicate that difference is statistically significant at ${ }^{*} P<0.01$ and ${ }^{* *} P=0.01-0.05$ 
GW treatment did not induce a significant increase in the length of the membrane-bound portion of the posterolateral and ventral flagella while, in contrast, GW-treated cells clearly showed an extension of the anterior and caudal flagella in their membrane-bound parts of up to 11.3 and $11.1 \mu \mathrm{m}$, respectively. These data clearly showed that the GW treatment affected the formation of these two flagella among the four types of flagella present in $G$. lamblia.

\section{Localization of GIPLK and definition of domains required for its localization in Giardia trophozoites}

A homology search in the Giardia database indicated an ORF (GL50803_104150) as the putative G. lamblia PLK, GIPLK. Amino acid sequences deduced from the ORF were aligned with those of human and Trypanosoma brucei PLKs (GenBank accession numbers NP_005021.2 and Tb927.7.6310, respectively), showing $31-34 \%$ identity (Additional file 3: Fig. S2). The
ORF was postulated to encode a protein of $\mathrm{pI}=8.8$, and a search of domains within this ORF using the Entrez program (http://www.expasy.org/) indicated that it contains a serine/threonine kinase domain (KD) at the amino-terminal portion (from amino acid residue no. 20 to 309). In addition, blocks of amino acids near the carboxyl terminus were proposed as the PBDs (amino acid residues no. 432-517 and 563640), which had been conserved in diverse PLKs [23]. Based on the alignment of GIPLK with other PLKs, Lys51 was suggested as a residue that initially receives phosphate from ATP, and Thr179 and Thr183 residues were proposed as target sites that are subsequently phosphorylated.

A plasmid, pGIPLK.neo, was constructed (Fig. 3a) and used to construct transgenic Giardia trophozoites expressing HA-tagged GIPLK. Western blotting of the resulting $G$. lamblia extracts confirmed the expression

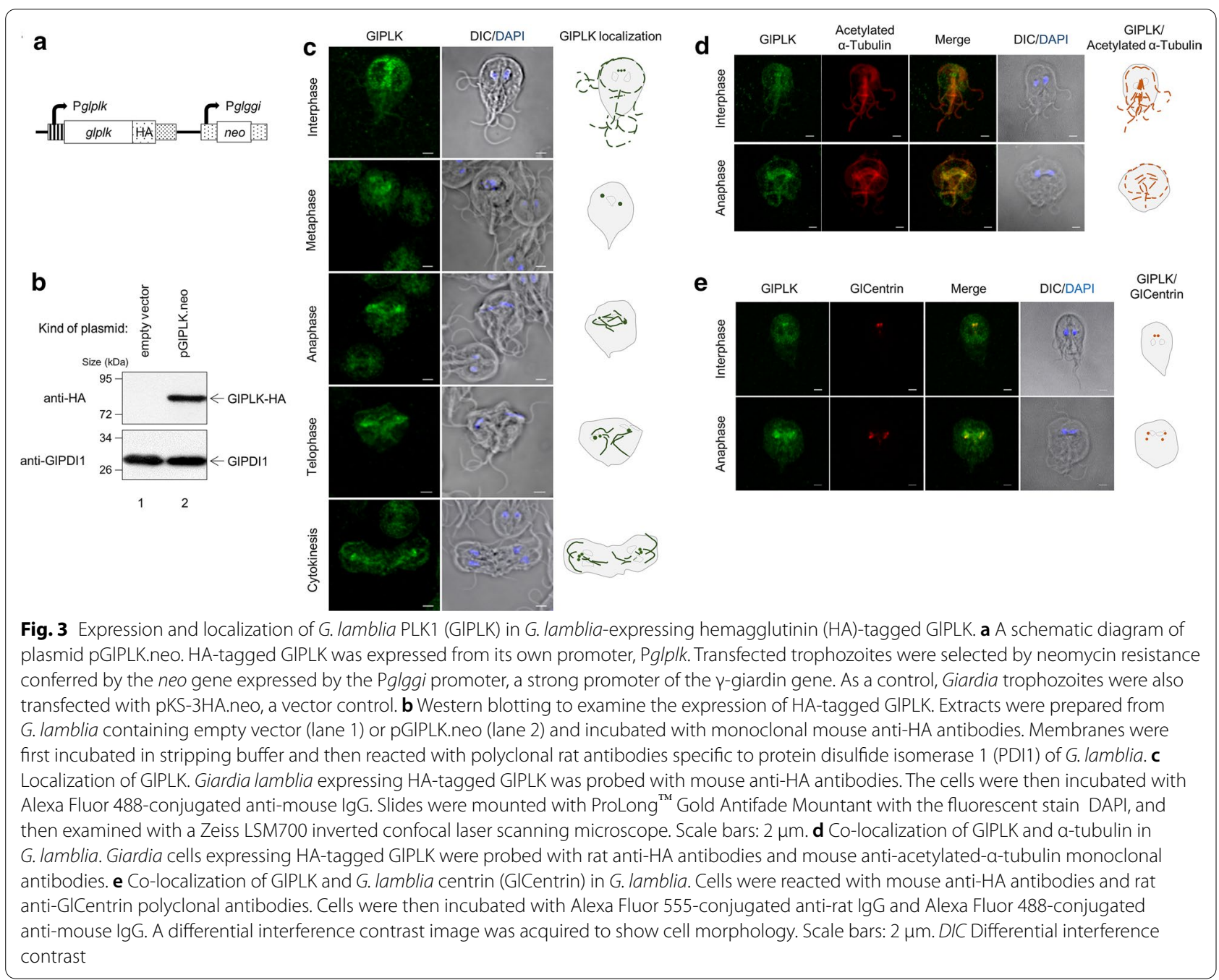


of HA-tagged GlPLK as an immunoreactive band with a molecular weight of $80 \mathrm{kDa}$ (Fig. 3b). In contrast, the extracts of G. lamblia carrying the vector control, pKS3HA.neo, did not produce any immunoreactive bands in the same analysis. Western blotting of the same membrane with anti-GlPDI1 antibodies [24] served as a loading control for the total amount of protein in the extracts used for this assay.

The localization of GIPLK was determined using Giardia expressing HA-tagged GIPLK (Fig. 3c). GIPLK was found in the basal bodies, flagella, axonemes, an adhesive disc and median bodies of Giardia trophozoites at interphase. Localization at the basal bodies was maintained in the dividing cells (cells at metaphase, anaphase and telophase), as well as at cytokinesis. In cells at anaphase, GIPLK was also present in the mitotic spindles and axonemes of the dividing cells.

To confirm the localization of GlPLK, Giardia cells expressing HA-tagged GIPLK were double-stained for GIPLK and microtubules (MTs) using anti-HA and antiacetylated- $\alpha$-tubulin antibodies, respectively (Fig. 3d). In Giardia cells at interphase and anaphase, GIPLK was found together with MTs in the basal bodies, axonemes, median bodies and flagella. Giardia cells at anaphase also demonstrated the co-localization of GIPLK with MTs in the mitotic spindles present between two separated groups of basal bodies.

Basal bodies serve as the MT-organizing center (MTOC) in G. lamblia [25], and the MTOC can be observed by staining for its marker, centrin. Additional IFAs for Giardia expressing HA-tagged GIPLK were performed using antibodies against HA and G. lamblia centrin (GlCentrin) (Fig. 3e). These double-stained Giardia cells clearly showed the co-localization of GIPLK and GlCentrin during cell division as well as at interphase.

As mentioned above, GIPLK comprises two regions: the KD and two PBDs (Fig. 4a). The region between the KD and PBDs was named the linker. To examine whether the KD and/or PBDs play a role in GIPLK localization, we constructed two plasmids, pGIPLKKDL.neo and pGIPLKPBD.neo, that expressed the KD linker and the PBDs of GIPLK, respectively. Western blotting using anti-HA antibodies revealed the expression of the truncated GIPLK proteins, KD linker and PBDs, in Giardia trophozoites in the form of immunoreactive bands with a molecular weight of 60 and $40 \mathrm{kDa}$, respectively (Fig. 4b). On the other hand, Giardia carrying the vector plasmid pKS-3HA.neo did not show any immunoreactive bands.

Giardia lamblia cells carrying pGIPLKKDL.neo were double-stained with anti-HA and anti-acetylated- $\alpha-$ tubulin antibodies or with anti-HA and anti-GlCentrin antibodies (Fig. 4c) in order to observe whether this truncated GlPLK-KDL was correctly localized in mitotic spindles and basal bodies, respectively. In interphase Giardia cells, double staining with antibodies against acetylated- $\alpha$-tubulin and HA resulted in the labeling of flagella, axonemes, a median body and basal bodies $(n=5)$. In contrast, GlPLK-KDL was not found in the mitotic spindles of the dividing Giardia at anaphase $(n=5)$. Double staining of G. lamblia cells carrying pGlPLKKDL.neo with anti-HA and anti-GlCentrin antibodies revealed the co-localization of these two proteins in basal bodies [cells at metaphase $(n=2)$ and cells at anaphase $(n=6)]$. However, the relative position of these structures with DAPI-stained nuclei indicated an incorrect localization of the basal bodies, as presented in an extended view.

In contrast, Giardia cells expressing truncated GIPLKPBD demonstrated the same pattern of co-localization with the full-length GIPLK with respect to $\alpha$-tubulin (Fig. 4d). In addition to being found in the flagella, axonemes and median bodies of the interphase cells $(n=3)$, GlPLK-PBD was found at the mitotic spindles in dividing Giardia cells $(n=7)$. Double staining of Giardia cells expressing GIPLK-PBD revealed the co-localization of this protein with GlCentrin in basal bodies $(n=7)$. Extended views of these dividing cells indicated that double-stained basal bodies positioned themselves in the correct positions for mitosis. These results suggest that the PBD of GIPLK is required for GIPLK localization in mitotic spindles and the correct positioning of basal bodies during Giardia cell division.

\section{Effect of GIPLK knockdown on cell division and flagella biogenesis in G. lamblia}

To define the role of this putative GIPLK in G. lamblia, we designed an anti-glplk morpholino to block the translation of glplk mRNAs (Table 1). A control morpholino (non-specific oligomer) was also synthesized and transfected by electroporation into G. lamblia trophozoites carrying pGIPLK.neo (Table 2). When the cells were harvested at various time-points, ranging from 12 to $48 \mathrm{~h}$, and analyzed for GIPLK inhibition, the cells at $24 \mathrm{~h}$ post-transfection demonstrated a maximal inhibition of GIPLK expression (data not shown). However, we chose the cells harvested at $18 \mathrm{~h}$ after transfection for further studies (Fig. 5a). In cells treated with an anti-glplk morpholino, the amount of HAtagged GIPLK at $18 \mathrm{~h}$ post-transfection had decreased to $59.5 \%$ of that in cells treated with the control morpholino $(P=0.0003)$. In addition, these extracts were examined to determine their intracellular GIPLKHA and GIPLK levels by western blotting using antiGlPLK antibodies. In cells treated with an anti-glplk morpholino, the amounts of GIPLK-HA and GIPLK at 


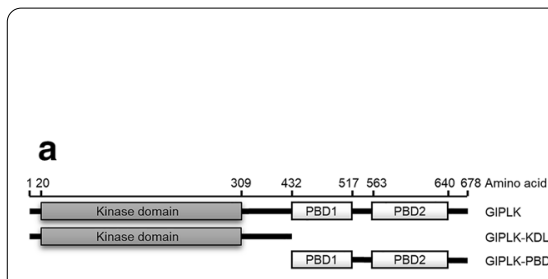

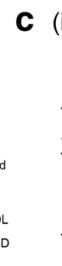
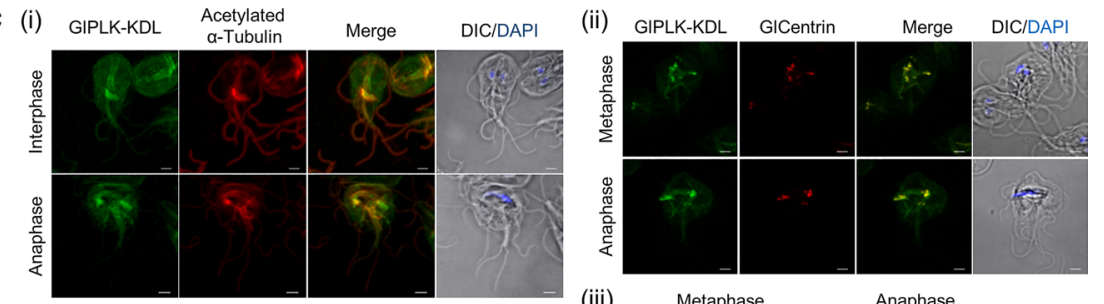

b

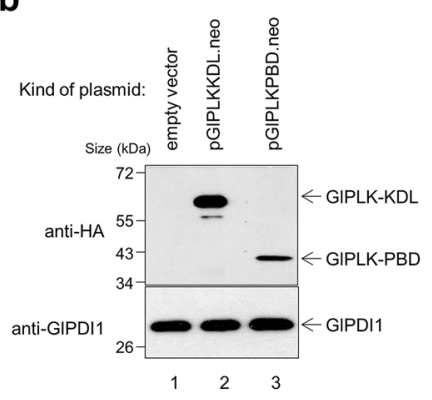

d

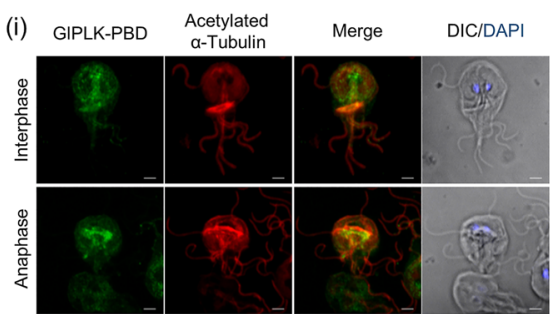

(iii)

Metaphase

Anaphase

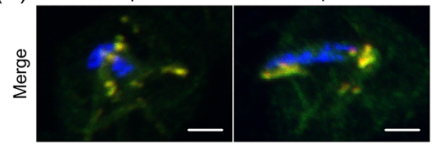

(ii) GIPLK-PBD GICentrin Merge DIC/DAPI

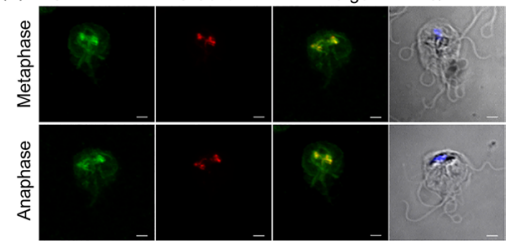

(iii)

Metaphase

Anaphase

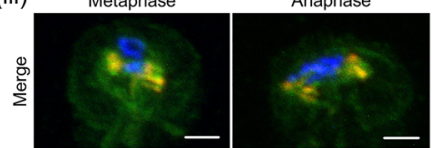

Fig. 4 Expression and localization of truncated GIPLKs in G. lamblia. a A schematic diagram of plasmids pGIPLKKDL.neo and pGIPLKPBD.neo. Two truncated GIPLK proteins are expressed in an HA-tagged form from their own promoter, Pglp/k. Plasmid pGIPLKKDL encodes GIPLK with the KD and linker region, whereas PGIPLKPBD contains DNA coding for the polo-box domains (PBDs) of GIPLK. Plasmid pKS-3HA.neo was transfected into Giardia trophozoites as a control. b Western blotting to examine the expression of HA-tagged truncated GIPLKs. Extracts were prepared from G. lamblia containing empty vector (lane 1), pGIPLKKDL.neo (lane 2), or pGIPLKPBD.neo (lane 3), and incubated with monoclonal mouse anti-HA antibodies. Membranes were incubated in stripping buffer, and then reacted with polyclonal rat antibodies specific to GIPDI1. c Co-localization of GIPLK-KDL with a-tubulin (i) or GICentrin (ii, iii). Giardia lamblia cells expressing HA-tagged truncated GIPLK-KDL were probed with rat anti-HA antibodies and mouse anti-acetylated-a-tubulin monoclonal antibodies. Otherwise, these cells were reacted with rat anti-GlCentrin polyclonal antibodies instead of anti-acetylated-a-tubulin monoclonal antibodies. Panel iii is an extended view of panel ii. Incorrectly positioned basal bodies are indicated with white arrows. $\mathbf{d}$ Co-localization of GIPLK-PBD with a-tubulin (i) or GICentrin (ii, iii). G. lamblia cells expressing HA-tagged truncated GIPLK-PBD were probed with rat or mouse anti-HA antibodies along either with mouse anti-acetylated-a-tubulin antibodies (i), or rat anti-GICentrin polyclonal antibodies (ii, iii), respectively. Panel iii is an extended view of panel ii. The cells were then incubated with Alexa Fluor 488-conjugated anti-rat lgG and Alexa Fluor 568-conjugated anti-mouse lgG (a-tubulin co-localization) or Alexa Fluor 555-conjugated anti-rat IgG

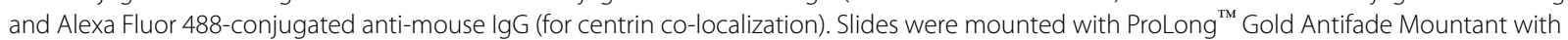
DAPI, and then examined with a Zeiss LSM700 inverted confocal laser scanning microscope. A differential interference contrast image was acquired to show cell morphology. Scale bars: $2 \mu \mathrm{m}$

$18 \mathrm{~h}$ post-transfection had decreased to 56 and $55 \%$ of those in cells treated with the control morpholino, respectively.

The effect of GlPLK knockdown on cell division was determined based on the nuclear phenotypes, which included the number of nuclei and the condensation of the DNA in the cells (Fig. 5b). The percentage of cells with two normally positioned nuclei decreased from 98.7 to $90.7 \%$. Among these cells, the number of cells showing nuclear condensation increased from 0.4 to $4.6 \%$. The percentages of cells with one nucleus, two nuclei in abnormal position, three nuclei and four nuclei were slightly increased in cells treated with an antiglplk morpholino, without any statistical significance.
Only the percentage of cells with more than four nuclei showed a statistically significant increase in anti-glplk morpholino-treated cells $(P=0.02)$.

The effect of GIPLK depletion on the DNA ploidy of Giardia cells was also determined by flow cytometric analysis (Fig. 5c). Cells treated with control morpholino for 6 or $18 \mathrm{~h}$ showed a similar proportion of cells in the G1/S and G2/M phases (19 and 79\%, respectively). In cells treated with anti-glplk morpholino, the percentage of G2/M-phase cells increased to $84 \%$ after $6 \mathrm{~h}$ posttransfection. In contrast, a greater number of cells treated with anti-glplk morpholino for $18 \mathrm{~h}$ were present in the G1/S phase (25\%), as compared with the control cells (17\%). The percentage of G2/M-phase cells decreased 

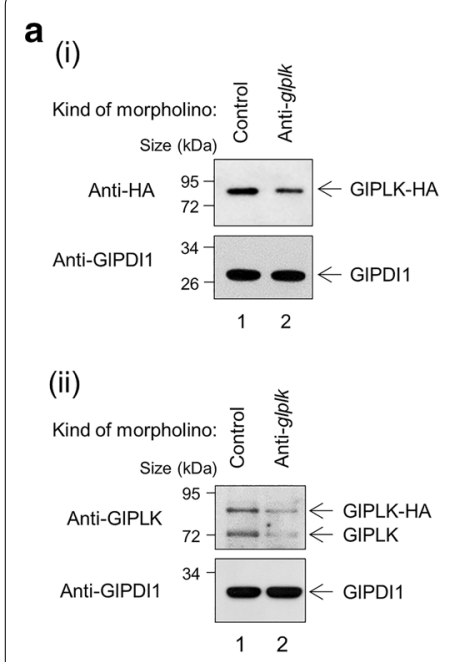
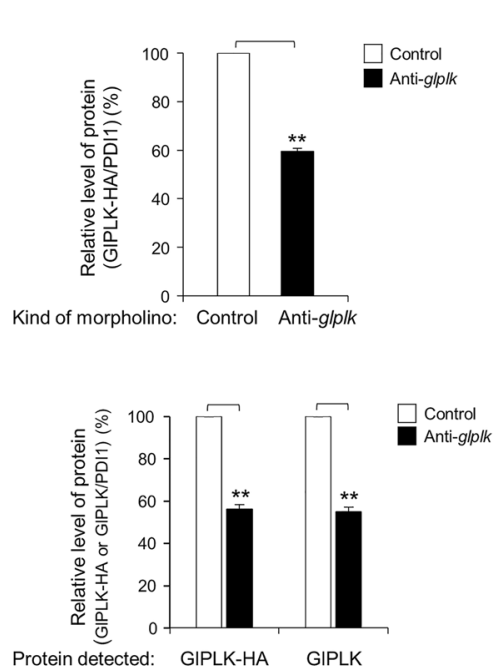

Protein detected: GIPLK-HA GIPLK
C (i)

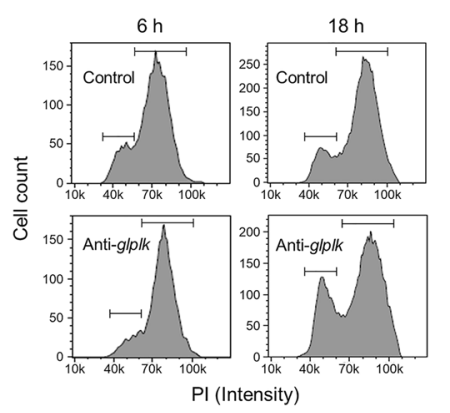

(ii)

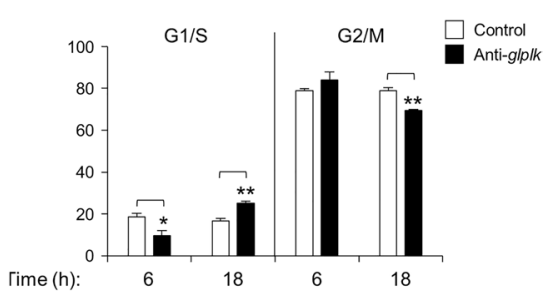

d

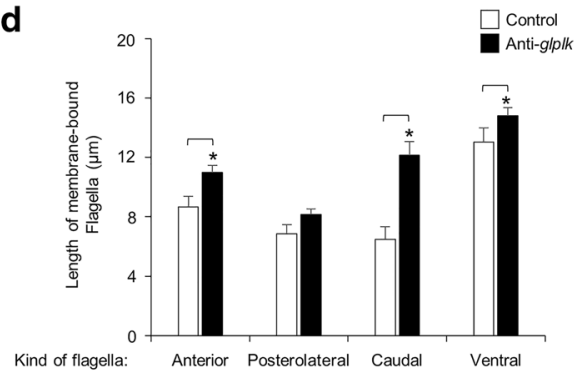

Position of nuclei: $\quad$ Normal Abnormal

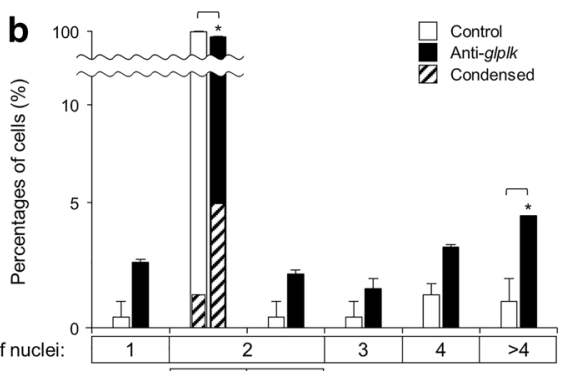

Fig. 5 Effect of morpholino-mediated GIPLK knockdown in cell division and flagella formation in G. lamblia. Giardia trophozoites
expressing HA-tagged GIPLK were collected at $18 \mathrm{~h}$ after electroporation with control (open bars) or anti-glplk morpholino (closed bars). a Morpholino-mediated GIPLK knockdown in G. lamblia. (i) Western blot analysis using anti-HA or anti-GIPDI1 antibodies, (ii) western blot analysis using anti-GIPLK or anti-GIPDI1 antibodies. The relative expression of HA-tagged GIPLK (i) or/and endogenous GIPLK (ii) in extracts of cells treated with an anti-glplk morpholino compared with the expression in the control cells is presented as a bar graph. $\mathbf{b}$ Effects of morpholino-mediated GIPLK knockdown on the nuclear phenotypes of G. lamblia. The cells transfected with control (open bars) or an anti-glplk morpholino (closed bars) were maintained for $18 \mathrm{~h}$ prior to staining with Giemsa solution. At least 300 cells were examined for the number and position of nuclei under each condition using an Axiovert 200 microscope. Among the cells with two nuclei in the normal position, the number of cells showing nuclear condensation was also recorded (hatched bars). c Effects of morpholino-mediated GIPLK knockdown on the ploidy of their DNA of G. lamblia. The cells transfected with control (open bars) or an anti-glplk morpholino (closed bars) were maintained for $18 \mathrm{~h}$. After being stained with Pl, the ploidy of their DNA was analyzed by flow cytometry. $\mathbf{d}$ Effects of morpholino-mediated GIPLK knockdown on flagella formation in G. lamblia. Flagella length was measured in 35 cells per condition. Data are presented as an average of three independent experiments. Asterisks indicate that the difference is statistically signiicant at ${ }^{*} P=0.01-0.05$ and ${ }^{* *} P<0.01$, respectively

to $69 \%$ after $18 \mathrm{~h}$ post-transfection of anti-glplk morpholino, compared 79\% in control cells.

GIPLK depletion also resulted in the formation of Giardia trophozoites with longer flagella (Fig. 5d). The length of the membrane-bound portion of the caudal flagella in cells treated with an anti-glplk morpholino increased to $12.1 \mu \mathrm{m}$ compared to $6.5 \mu \mathrm{m}$ in the control morpholino cells $(P=0.003)$. GlPLK-depleted cells also showed extension of the anterior and ventral flagella in their membrane-bound parts of up to 11.0 and $14.8 \mu \mathrm{m}$ compared to 8.7 and $13.0 \mu \mathrm{m}$, respectively, in control cells.

\section{Expression pattern of GIPLK at G1/S and G2/M phase of the Giardia cell cycle}

As human PLK1 is highly expressed during mitosis [26], we examined whether GIPLK expression varies in a phase-dependent manner. Giardia cells were treated with nocodazole to prepare G2/M-phase cells (91\%) or sequentially with nocodazole and aphidicolin to acquire G1/S-arrested cells (86\%). The stage of the resulting Giardia cells carrying pGIPLK.neo was confirmed by flow cytometry (Additional file 4: Fig. S3A). Control Giardia trophozoites treated with $0.01 \%$ DMSO were 
found to be a mixture of G1/S- and G2/M-phase cells, G2/M-phase cells being the dominant cell type (78\%).

Western blotting of these cell extracts using anti-HA antibodies demonstrated an increased amount (1.5-fold) of GlPLK in G2/M-phase and interphase cells in comparison with G1/S-phase cells (Additional file 4: Fig. S3B). The immunoreactive band was absent from the extracts prepared from Giardia cells carrying pKS-3HA.neo. Western blotting of the same blot using anti-GlPDI1 antibodies served as a loading control.

Increased expression of the glplk transcript was also examined using an alternative method, quantitative reverse transcription (RT)-PCR (Additional file 4: Fig. $\mathrm{S} 3 \mathrm{C})$. The relative level of glplk transcripts to glactin transcripts remained increased (2.4-fold) in G2/M-phase cells compared to $\mathrm{G} 1 / \mathrm{S}$-phase cells $(p=0.01)$. To monitor the phases of our samples, the assays included two G1/S phase marker genes encoding histone $\mathrm{H} 3$ and histone $\mathrm{H} 4$, which showed a decreased expression in the G2/M-phase cells [5]. In addition, the transcript level of Gly-giardin was measured in these cells and found to show increased expression in G2/M phase, as expected [16].

\section{Subcellular localization of GIPLK in G. lamblia}

In order to function properly during mitosis, PLK1 should be localized to specific sites through differential interaction with various scaffold proteins [23]. The nucleus is one of the subcellular locations where PLK1 localizes in the G2 phase [27].

Giardia extracts were prepared from Giardia cells expressing HA-tagged GIPLK at interphase, the G1/S phase and the G2/M phase, and then further divided into cytoplasmic and membrane fractions, which may include nuclear fractions. These extracts were analyzed by western blotting using anti-HA antibodies (Additional file 5: Fig. S4). In addition, extracts were evaluated for G. lamblia glyceraldehyde 3-phosphate dehydrogenase (Gl50803_6687; GlGAP1), G. lamblia centrin (Gl50803_104685; GlCentrin) and G. lamblia centromeric histone H3 (GL50803_20037; GlCenH3) as markers for cytoplasmic, membrane and nuclear proteins, respectively. Because the amino acid sequence alignment of the three histone H3 proteins of G. lamblia demonstrated $33-46 \%$ identity among them, it is unlikely that anti-GlCenH3 reacts with the other two histone H3 proteins. Even though there is some cross-reactivity of these antibodies against the other histone $\mathrm{H} 3$ proteins, it did not interfere with this experiment in that all three hisone H3 proteins are located in the nuclei of G. lamblia [20].

GIPLK was found in both the cytoplasmic and membrane fractions in all examined phases. As expected, GlGAP1 was mainly present in the cytoplasmic fraction, and GlCentrin and GlCenH3 were found only in the membrane fraction.

Both G1/S- and G2/M-phase cells demonstrated GlPLK localization in the membrane fraction, and more GIPLK was found in the G2/M-phase cells than in the G1/S-phase cells. A constant amount of GlGAP1 was present in the cytoplasmic fraction of all examined phases, whereas more GlCentrin and $\mathrm{GlCenH} 3$ were found in the membrane fraction of the G2/M-phase cells than in the G1/S-phase cells.

\section{Expression and localization of phosphorylated GIPLK in G. lamblia}

In addition to the expression of GlPLK, GIPLK activity is important for its role in Giardia cell division; this role may be regulated by its activation status, possibly by phosphorylation. We examined whether GIPLK phosphorylation was modulated in a cell phase-dependent manner (Fig. 6a). Giardia cells carrying pGlPLK.neo were used to prepare the cell extracts at interphase, G1/S phase and G2/M phase, and then analyzed by western blotting using anti-phospho-PLK, anti-HA, anti-GlPLK and anti-GlPDI1 antibodies. In the western blot analysis with anti-phospho-PLK, both HA-tagged GIPLK and endogenous GlPLK were detected, and the amount of both proteins increased fivefold and 2.5-fold during the G2/M phase, respectively. In the same manner, western blotting using anti-GlPLK and anti-HA antibodies demonstrated more than a twofold increase in the expression of both HA-tagged GIPLK and endogenous GIPLK during the G2/M phase.

Giardia trophozoites were double-stained with antiHA and anti-phospho-PLK antibodies (Fig. 6b). Both anti-HA and anti-phospho-PLK antibodies stained basal bodies in the interphase and dividing cells. Localization of phospho-GIPLK in the cytoplasmic portion of anterior flagella, median bodies, and flagella tips was distinct. In dividing cells, phospho-GlPLK was also found at mitotic spindles, as with the HA-tagged GIPLK.

\section{In vitro autophosphorylation of GIPLK and identification} of critical amino acid residues for its autophosphorylation

The putative amino acid sequence of GIPLK indicates a serine/threonine $\mathrm{KD}$ at the amino terminus and two PBDs at the carboxyl terminus (Fig. 7a). Based on comparison with other PLKs, it was predicted that Lys51 is the primary binding site for ATP, and that the phosphate of Lys 51 is eventually transferred to Thr179 and Thr183 in the activation loop.

Kinase assays were also performed using recombinant GIPLK (rGlPLK), which was synthesized using in vitro transcription and translation systems, and expression was confirmed by western blotting with anti-c-Myc 


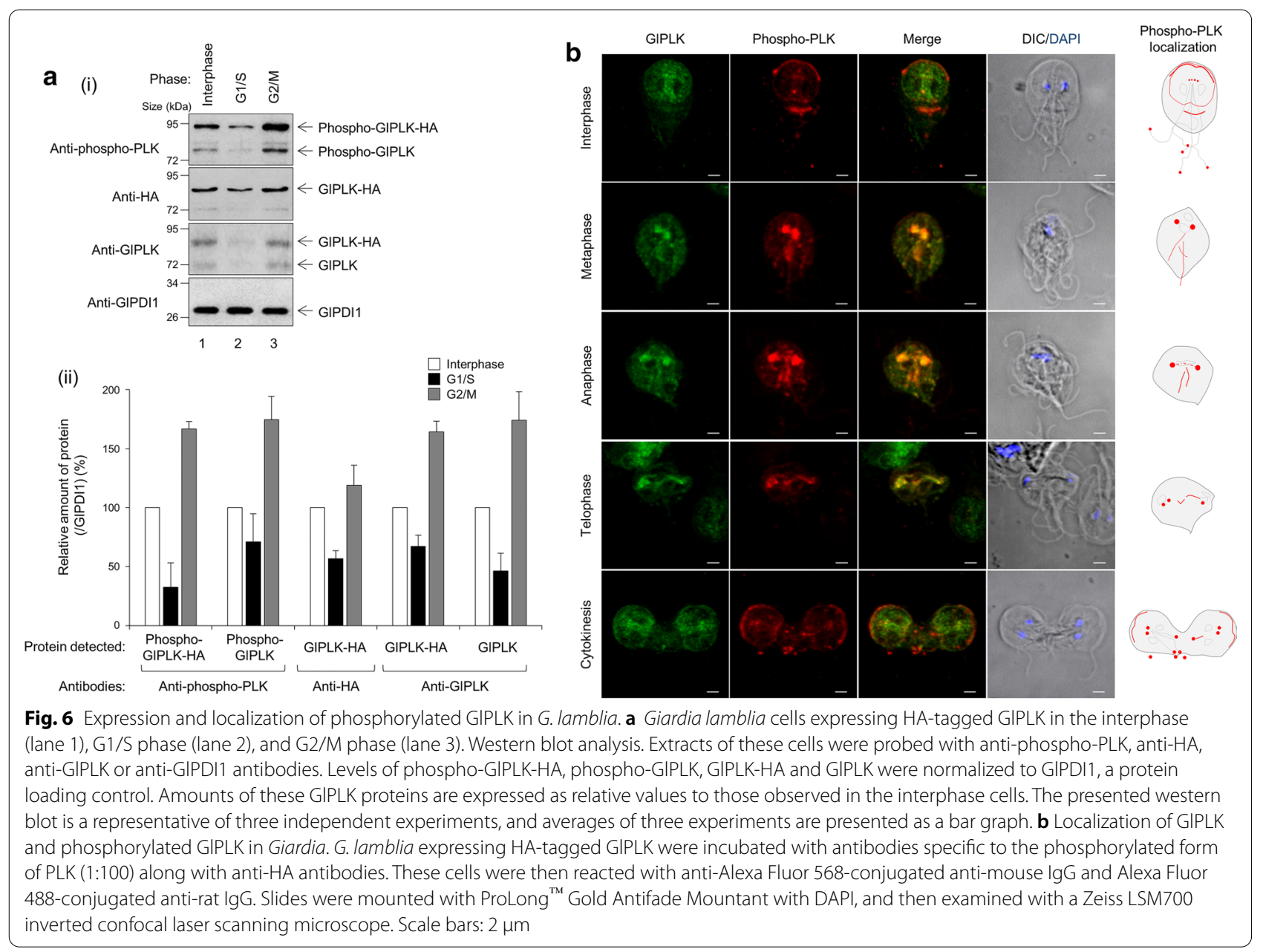

antibodies (Fig. 7b). Upon incubation with $\left[\gamma_{-}{ }^{32} \mathrm{P}\right] \mathrm{ATP}$, rGIPLK was radiolabeled due to autophosphorylation.

To define the amino acid residues that are critical for GIPLK autophosphorylation, several recombinant GIPLK proteins were synthesized using in vitro transcription/ translation systems and used for kinase assays (Fig. 7c). Specifically, the two putative phosphorylation sites were mutated to Ala, and the resulting mutant GIPLK proteins (GIPLKT179A and GIPLKT183A) were used for kinase assays. In an additional mutant GIPLK, the putative ATP binding site of Lys51 was mutated to Arg (GIPLKK51R). Both GIPLKT179A and GIPLKT183A proteins were autophosphorylated, although the efficiency of autophosphorylation was lower than that of wild-type GIPLK. When both Thr179 and Thr183 were mutated to Ala in GIPLK, the resulting protein exhibited a dramatic decrease in its autophosphorylation ability. Conversion of Lys51 to Arg abolished the autophosphorylation of rGIPLK. This result demonstrated that both Thr179 and Thr183 in the activation loop of GIPLK were phosphorylated. As expected, Lys51 of GlPLK was confirmed to serve as an ATP binding site.

\section{Role of GIPLK phosphorylation in cytokinesis and flagella biogenesis in G. Iamblia}

Subsequent experiments were performed to determine the physiological roles of GIPLK. Transgenic G. lamblia carrying pGlPLKK51R.neo was constructed. In addition, Giardia cells ectopically expressing mutant GIPLK (T179AT183A) were prepared. Western blotting demonstrated that the transgenic cells expressed HA-tagged GIPLK proteins (Fig. 8a).

The growth of various Giardia cells (ectopically expressing GIPLK, mutant GLPLKK51R, mutant GIPLKT178AT183A or carrying empty vector) was determined (Fig. 8b). The growth of Giardia cells overexpressing wild-type GIPLK was slightly affected when compared with that of the control cells. Interestingly, Giardia cells expressing mutant GIPLKs showed dramatic growth inhibition compared to those carrying the vector plasmid. 


\section{a}
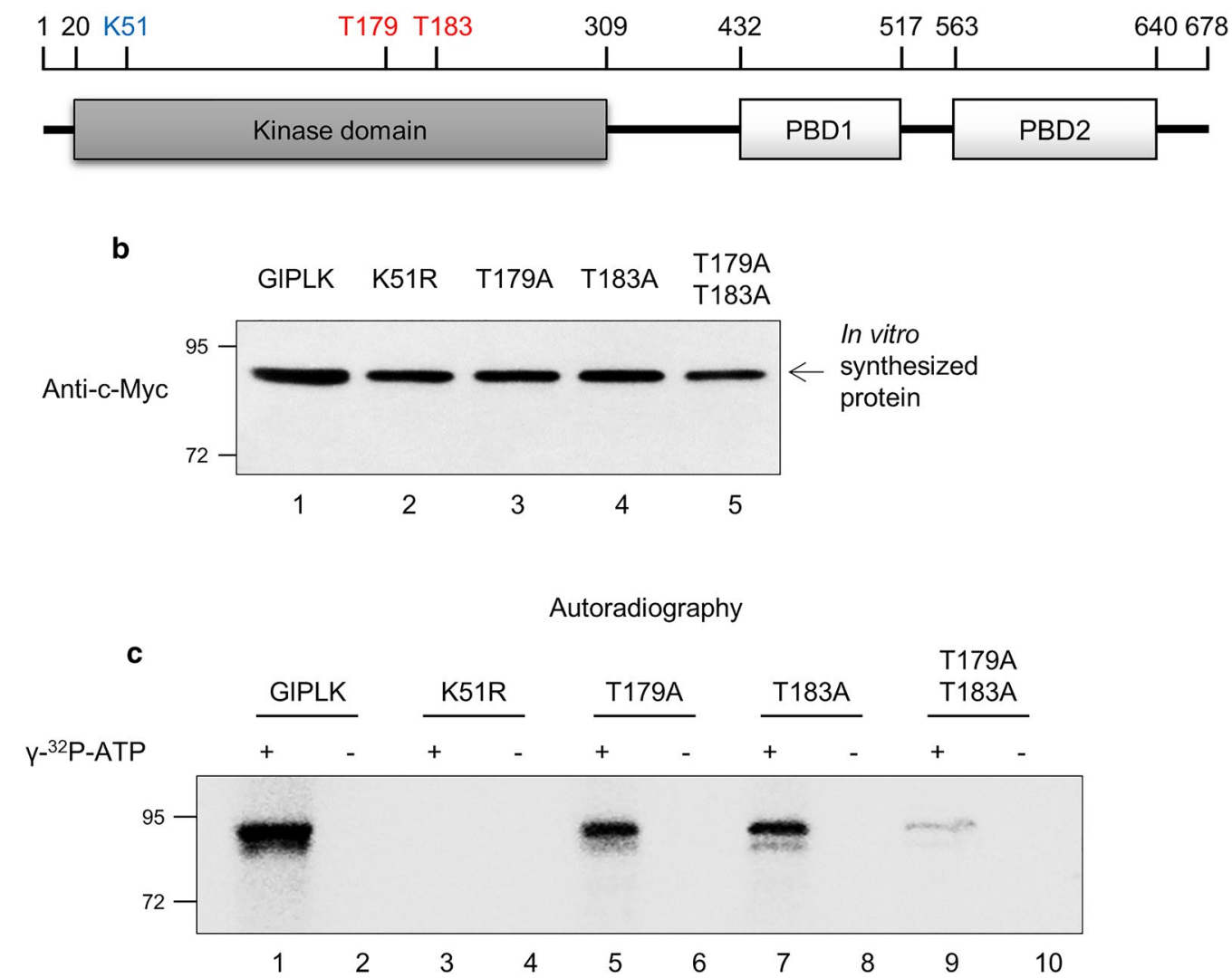

Fig. 7 In vitro autophosphorylation of wild-type and mutant GIPLKs. a A schematic diagram of GIPLK. Serine/threonine kinase (KD) and two polo-box domains (PBD1, PBD2) are indicated as boxes. Lys51 (K51) is suggested as a residue that initially receives phosphate from ATP, and two threonine residues (T179 and T183) are proposed as the target sites of subsequent phosphorylation. $\mathbf{b}$ Western blot of rGIPLK proteins synthesized in vitro using mouse anti-c-Myc antibodies (1:1000). c Phosphorylation of in vitro-synthesized GIPLKs. The c-Myc-tagged GIPLKs (wild-type GIPLK, K51R mutant GIPLK, and T179AT183A mutant GIPLK) were prepared in vitro and then used for kinase assays. GIPLK proteins were resuspended in $20 \mu \mathrm{l}$ kinase buffer in the presence of $2.5 \mu \mathrm{Ci}\left[\mathrm{Y}^{-}{ }^{32} \mathrm{P}\right]$ ATP. The kinase reactions were then subjected to $12 \%$ SDS-PAGE and visualized by autoradiography

These cells were then evaluated for their number of nuclei as described earlier (Fig. 8c). The majority of the control cells carrying vector plasmid and cells overexpressing wild-type GIPLK (98\%) had two nuclei in the correct position. The percentage of cells with two nuclei in the correct position were decreased to $88-89 \%$ when mutant GlPLKs were ectopically expressed. Among these cells, the number of cells showing nuclear condensation increased to 4.7 and $2.6 \%$ from 0.2 to $0.3 \%$ (control and GlPLK-overexpressing cells, respectively) in the case of Giardia expressing GLPLKK51R and GIPLKT179AT183A. In contrast to a slight increase in the number of cells with one nucleus, two abnormally located nuclei and three nuclei, the cells expressing mutant GlPLKs showed a dramatic increase in percentage of cells carrying four nuclei and more than four nuclei. These results indicate that Lys51, as well as two Thr residues (Thr179 and Thr183), in GlPLK may play a role in cell division in Giardia. GlPLK overexpression did not affect the length of flagella. In contrast, the ectopic expression of mutant GIPLK resulted in the extension of the lengths of three flagella, except for the posterolateral flagella (Fig. 8d). These data indicate that GIPLK plays a role in regulating flagella morphogenesis and cell cycle in Giardia and that GlPLK phosphorylation is critical for its in vivo function.

\section{Discussion}

Mammalian PLK is a multi-faceted kinase that controls several steps of the cell cycle [28]. In contrast to the presence of PLK paralogues in other systems, G. lamblia seems to have one PLK, the function of which was demonstrated in the present study with a chemical inhibitor of PLK (Additional file 2: Table S1; Figs. 1, 2) and an antiglplk morpholino (Fig. 5). 


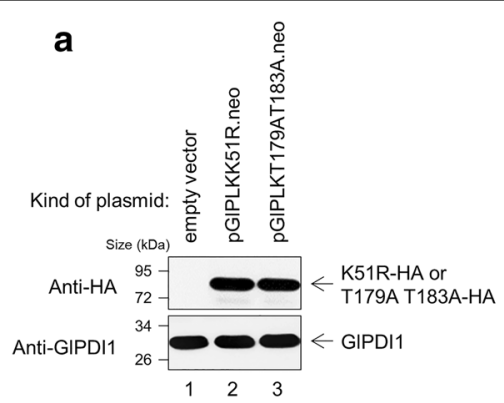

C

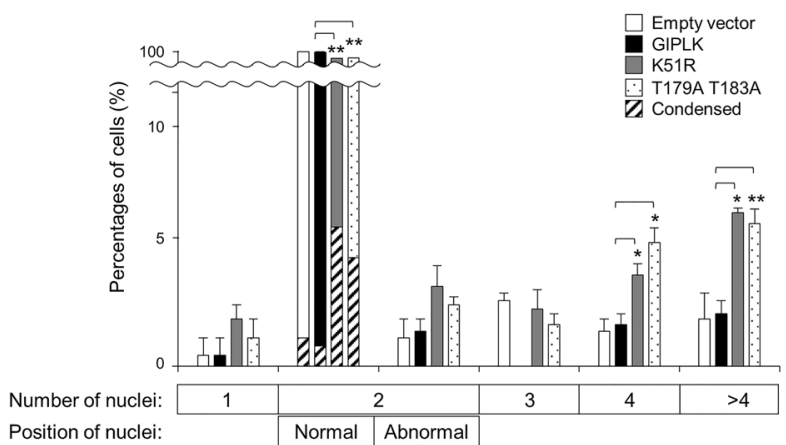

b

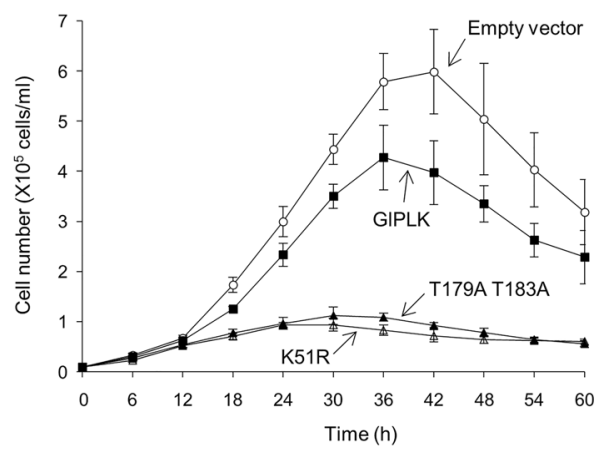

d

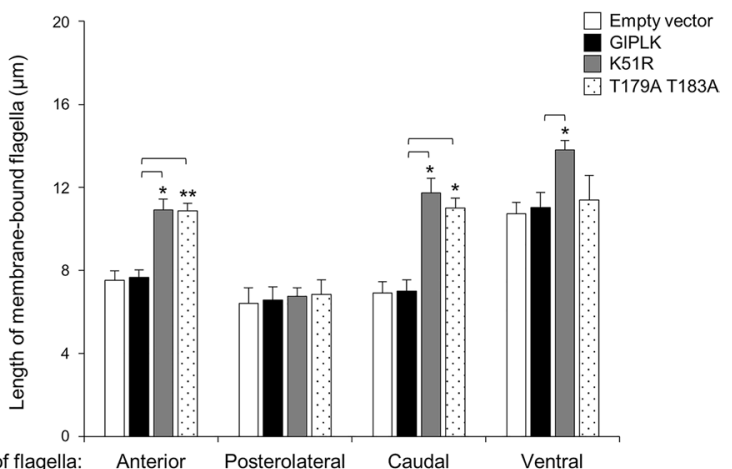

Fig. 8 Functional defects caused by ectopic expression of mutant GIPLKs in Giardia. Giardia carrying the empty vector (pKS-3HA.neo), and Giardia expressing wild-type GIPLK, K51R mutant GIPLK, or T178AT183A mutant GIPLK, were constructed by electroporation of the corresponding plasmid into Giardia trophozoites. a Western blot analysis showing the expression of HA-tagged mutant GIPLK proteins in Giardia. Cell extracts were prepared from Giardia carrying the vector plasmid (lane 1), Giardia expressing K51R mutant GIPLK (lane 2), or T178AT183A mutant GIPLK (lane 3), and reacted with anti-HA antibodies. b Growth curves of Giardia carrying the vector plasmid (open circles) or expressing wild-type GIPLK (closed squares), K51R mutant GIPLK (open triangles) or T178AT183A mutant GIPLK (closed triangles). The number of parasites per milliliter was determined using a hemocytometer. Each experiment comprised three cultures and was repeated three times using independently obtained transfected cells. c Effects of the ectopic expression of mutant GIPLKs on the nuclear phenotypes of G. lamblia. Various cells, Giardia carrying the vector plasmid (open bars) and Giardia expressing wild-type GIPLK (closed bars), K51R mutant GIPLK (gray bars) or T178AT183A mutant GIPLK (dotted bars), were stained with Giemsa solution. At least 300 cells were examined for the number and position of the nuclei under each condition using an Axiovert 200 microscope. Among the cells with two nuclei in the normal position, the number of cells showing nuclear condensation was also recorded (hatched bars). $\mathbf{d}$ Effects of the ectopic expression of mutant GIPLKs on flagella formation in G. lamblia. Flagella length was measured in 35 cells per condition. Data are presented as an average of three independent experiments. Asterisks indicate that the difference is statistically signiicant at ${ }^{*} P=0.01-0.05$ and ${ }^{* *} P<0.01$, respectively

Since the phenotype we monitored was the number of nuclei of GlPLK-inhibited and GlPLK-depleted Giardia trophozoites (Additional file 2: Table S1; Figs. 1a, 5b), these experiments only provide evidence for the role of GIPLK in cytokinesis, but not in other aspects of the cell cycle, such as centrosome maturation, kinetochore formation and mitotic spindle function. However, the increased percentage of cells with condensed DNA among the cells with two nuclei under these conditions (Figs. 1b, 5a) suggested that GIPLK plays an important role in mitosis similar to that of mammalian PLK1 [23]. This suggestion was further strengthened by IFAs showing the localization of GIPLK and phospho-GIPLK at basal bodies and mitotic spindles in dividing cells (Figs. 3c-e, 6b, respectively). GIPLK localization at basal bodies and mitotic spindles was confirmed by co-localization experiments using marker proteins (Fig. 3d, e).
The meaning of the results related to the effect of GIPLK inhibition on DNA ploidy of Giardia trophozoites (Fig. 1c) is difficult to interpret in that the arrested phases varied depending upon the treatment time with GW. At 6 and $12 \mathrm{~h}$ post- GW treatment, more Giardia trophozoites were found at the G2/M phase, whereas majority of cells were arrested at G1/S phase after $18 \mathrm{~h}$ and $24 \mathrm{~h}$ post-GW treatment. Therefore, it is premature to draw any conclusion on the role of GIPLK in a specific phase of the Giardia cell cycle. Considering the generation time for Giardia trophozoites, i.e. $4 \mathrm{~h}$, the results showing an increased number of cells at the G2/M phase at 6 and $12 \mathrm{~h}$ are more plausible. However, we cannot rule out the possibility that GIPLK also plays a role in the G1/S phase. Flow cytometric analysis on GlPLK-depleted Giardia using anti-glplk morpholino also demonstrated a similar pattern of time-dependent arrests even though degrees of 
arrests are less than those with GW treatment (Fig. 5c). In contrast to the restricted functions of PLK2, PLK3 and PLK5 in non-proliferating vertebrate cells [29], PLK1 and PLK4 are highly conserved. PLK1 is a multi-functional kinase involved in mitosis and cytokinesis, whereas PLK4 is known to function as a centriole assembly factor in the $S$ phase [30]. It is possible that GIPLK functions as a combined form of PLK1/PIK4.

Since the subcellular localization of PLK occurs via interactions with various scaffold proteins and is important for its functions in other systems [31], Giardia extracts were fractionated into cytoplasmic and membrane fractions in order to examine whether GIPLK is localized in a certain fraction (Additional file 5: Fig. S4). GIPLK was found in both fractions under any of the conditions. Most notably, IFAs showed no evidence of the nuclear localization of GIPLK (Figs. 3c, 6b). However, the subcellular fractionation assay showed that GIPLK was present in the membrane fractions, which contained the nuclear protein marker centromeric histone $\mathrm{H} 3$ and centrin localized at basal bodies (Additional file 5: Fig. S4). Interestingly, in the membrane fractions, the amount of GIPLK increased in the G2/M phase compared to that in the G1/S phase. However, our data cannot provide any evidence for the nuclear localization of GIPLK. The nuclear localization signal (NLS) and destruction box (D-box) were not observed in the amino acid sequence of GIPLK, whereas PLK1 has canonical sequences for the NLS and D-box [32]. Studies have shown that PLK1 SUMOylation is involved in its nuclear localization [33, 34]. A putative SUMO interaction sequence and a target sequence for SUMO were found in GIPLK using a SUMOylation prediction program (GPS SUMP 1.0). The absence of the D-box in cyclin B, AK and PLK of G. lamblia indicates a regulatory mechanism other than ubiquitin-mediated degradation [35]. Therefore, it will be interesting to study how the SUMOylation of GlPLK affects its localization and function during the cell cycle of G. lamblia.

In mammalian systems, PLK1 interacts with other proteins via its PBDs, and these interactions are critical for the spatial and temporal function of PLKs as they control their subcellular localization [36]. The roles of KD and PBD in the localization of GIPLK were examined using Giardia ectopically expressing truncated GIPLK proteins (Fig. 4). While the localization of GIPLK-PBD was similar to that of full-length GIPLK (Figs. 3c, 4d), the localization of GIPLK-KDL at mitotic spindles was not detected (Fig. 4c). Even though GIPLK-KDL was found together with GlCentrin at basal bodies, these double-stained basal bodies were misplaced in dividing cells, suggesting that GIPLK may be required for the proper function of basal bodies during cell division. Interestingly, the median bodies in trophozoites expressing truncated GIPLKs showed stronger labeling than those expressing full-length GlPLK.

When Giardia cells at different phases of the cell cycle were prepared by chemical treatment, both real-time PCR and western blotting showed upregulated GlPLK expression in cells in the G2/M phase compared with cells in the G1/S phase (Additional file 4: Fig. S3). An increased amount of phospho-GlPLK was also detected during the G2/M phase, as shown by western blotting using anti-phospho-PLK antibodies (Fig. 6a). In humans, the level of PLK1 is at its peak at metaphase [26]. A study using counterflow centrifugal elutriation of Giardia cells revealed a twofold increase in $g l p l k$ gene expression in the G2/M phase [5].

GIPLK autophosphorylation has been demonstrated in vitro using rGlPLK synthesized in vitro (Fig. 7c). Mutagenesis of GlPLK and kinase assays using the mutant rGIPLKs confirmed that Lys51 is a critical residue that receives phosphate from ATP. Two putative phosphorylation residues, Thr179 and Thr183, play a complementary and redundant role, based on the observation that phosphorylation was dramatically affected only when both of the residues were mutated.

These situations are more complex in vivo because PLK1 phosphorylation can occur in spatial and temporal modes. This phosphorylation depends upon the correct localization to the site at which the target protein is present and on the binding of the target proteins to the PBD of PLK1 [37]. When mammalian PLK1 is phosphorylated by aurora kinase (AK) A, mitosis is initiated in the cells [38]. In addition, cyclin B-CDK1-dependent phosphorylation of aurora borealis is a prerequisite for PLK activation [39]. GlAK was found in basal bodies (in interphase and dividing cells) and mitotic spindles (in dividing cells), and AK inhibition resulted in a defect in cytokinesis [24, $25,40]$. These results suggested that GIPLK may function together with GlAK during the cell cycle of G. lamblia. Moreover, an interaction between these two kinases was observed via co-immunoprecipitation (Kim et al., unpublished results).

The role of GIPLK was further confirmed by ectopically expressing mutant GIPLK in Giardia trophozoites (Fig. 8b, c). In addition to cytokinesis, the expression of mutant GIPLK proteins (K51R and T179AT183A) inhibited the growth of Giardia trophozoites, indicating that GIPLK affects cell division. However, the expression of wild-type GIPLK mildly affected cell growth and did not exert any effect on cytokinesis in G. lamblia. This result demonstrated that the amino acid residues critical for GlPLK phosphorylation are also important for GlPLK function in vivo. 
Lastly, we wish to address the effect of the GIPLK defect on flagellar homeostasis. While the cytoplasmic portion of the flagella was not affected, both GW-mediated and morpholino-mediated depletion of GIPLK resulted in the extension of the membrane-bound portion of the flagella in Giardia (Figs. 2, 5d). Interestingly, the membranebound regions of the caudal and anterior flagella were dramatically extended, whereas those of the posterolateral flagella were not significantly affected. The differential effect of GIPLK defects on the four types of flagella may be derived from the pattern of the redistribution of the flagella during Giardia cell division in which the posterolateral and ventral flagella are inherited from mother cells, whereas the other two flagella are newly synthesized [41]. The role of GIPLK in the homeostasis of flagella formation could be implied from its localization pattern in Giardia trophozoites during interphase, wherein it shows localization to the flagella and axonemes (Fig. 3c).

Phosphorylation of GIPLK is essential for its function in regulating flagella length, as Giardia cells ectopically expressing mutant phosphorylation-negative GIPLK proteins also showed extended flagella (Fig. 8d). It is interesting that the localization of phospho-GIPLK was distinct in the cytoplasmic portion of the anterior flagella and flagella tips (Fig. 6b). Another labeled structure found in these IFAs is median bodies, whose function and biogenesis are not clear (Figs. 3c, d, 4c, d, 5b). However, the median body is postulated as a reservoir for MT and a regulator of MT homeostasis. Localization of GIPLK to basal bodies, which function as an MTOC, indicated that GIPLK might play a role in MT nucleation. A previous study demonstrated that the depletion of the $\gamma$-tubulin ring complex $(\gamma$-TuSC) affects MT nucleation, resulting in the shortening of the flagella [18]. Overexpression of dominant-negative mutant kinesin-13, a motor protein, resulted in Giardia with longer flagella and defective mitotic spindles [42]. Core machineries for flagella biogenesis and control in Giardia have been investigated, revealing the presence of intraflagellar transport (IFT)mediated assembly [43]. The function of G. lamblia kinesin-13 at flagella pores results in the disassembly of flagella, as demonstrated by live-cell imaging and mathematical modeling of the conserved components of IFT and kinesin-13 [44]. IFT-mediated assembly and kinesinmediated disassembly are key processes in controlling the length of the flagella or cilia in Chlamydomonas or Tetrahynema thermophiles $[45,46]$. Our study added GIPLK to the list of components involved in the control of interphase flagellar length. In addition, flagella biogenesis has been reported to be an important factor for the cytokinesis of Giardia instead of actin-myosin-mediated mechanisms [6]. Interestingly, flagella shortening occurred in Giardia trophozoites in which expression of one of the
198 never-in-mitosis A (nimA)-related kinase (Nek) in Giardia, Nek8445, was depleted [47]. These studies suggest that GIPLK modulates flagella biogenesis via interaction with and/or modification of these proteins.

\section{Conclusions}

In this study, we demonstrated that G. lamblia has one PLK, which functions in the cell cycle and in flagella formation, as revealed by inhibitor-mediated and morpholino-mediated inhibition. We also demonstrated that the phosphorylation of GIPLK plays an important role in cell growth, cytokinesis and flagella biogenesis in Giardia.

\section{Abbreviations}

AK: Aurora kinase; CDK1: Cyclin-dependent kinase 1; GICENH3: Giardia lamblia centromeric histion H3; GICentrin: Giardia lamblia centrin; GIGAP1: Giardia lamblia glyceraldehyde 3-phosphate dehydrogenase; GIPLK1: Giardia lamblia PLK1; GW: PLK-specific inhibitor GW843286X; KD: N-terminal kinase domain; MT: Microtubules; MTOC: Microtubule-organizing center; PBD: Polo-box domain; PLK: Polo-like kinase.

\section{Supplementary Information}

The online version contains supplementary material available at https://doi. org/10.1186/s13071-021-04687-5.

Additional file 1: Figure S1. Effects of the PLK inhibitor GW843682X (GW) on Giardia growth. Growth inhibition of G. lamblia by GW. The numbers of Giardia trophozoites were counted using a hemocytometer 24-h posttreatment with various concentrations of GW $(5-15 \mu \mathrm{M})$.

Additional file 2: Table S1. Percentages of cells with different nuclei number upon GW treatment.

Additional file 3: Figure S2. Sequence alignment of putative G. lamblia PLK amino acids (GL50803_104150) with those of Trypanosoma brucei (Tb927.7.6310) and human (NP_005021.2). Identical residues are indicated with asterisks, whereas homologous residues are represented with dots. The serine/threonine kinase domain at the amino-terminal is denoted by a shaded box. Two blocks of amino acids near the carboxyl terminus were proposed to be polo-box domains. The activation domain (T-loop) of the kinase domain is underlined. A lysine $(K)$ in the amino-terminus region is suggested as a residue that initially receives phosphate from ATP, and two threonine residues in the T-loop are proposed as target sites of subsequent phosphorylation (indicated by bold letters).

Additional file 4: Figure S3. Expression of GIPLK in synchronized Giardia cells. a Flow cytometric analysis of Giardia trophozoites carrying PGIPLK. neo treated with $0.01 \%$ DMSO (interphase), trophozoites arrested with $100 \mathrm{nM}$ nocodazole for $3 \mathrm{~h}$ (G2/M), and trophozoites treated with $100 \mathrm{nM}$ nocodazole for $3 \mathrm{~h}$, followed by incubation with $6 \mu \mathrm{M}$ aphidicolin for $6 \mathrm{~h}$ (G1/S). b (i) Western blotting of synchronized cells using anti-HA antibodies. Lanes: 1 Giardia carrying the empty vector, 2-4 Giardia carrying pGIPLKHA.neo; lanes: 2 interphase cells, 3 G1/S-phase cells, 4 G2/M-phase cells. The amount of GIPDI 1 was also monitored in these cells using antiGIPDI1 antibodies. (ii) Relative levels of GIPLK to GIPDI1 are expressed as a bar graph. c Real-time quantitative assay. The mRNA quantity in the cDNA samples was normalized using the glactin transcript levels. Data are presented as the average of three independent experiments. ${ }^{*} P=0.01-0.05$.

Additional file 5: Figure S4. Subcellular fractionation of GIPLK in G. lamblia. Giardia carrying pGIPLK.neo was used to perform subcellular protein fractionation experiments. Both cytoplasmic and membrane protein fractions were prepared from interphase, G1/S-phase and G2/M-phase cells via sequential treatment with hypotonic and high-salt buffer. The amount 
of HA-tagged GIPLK in the extracts was monitored using anti-HA antibodies. The amount of GIGAP1, a cytoplasmic marker, was also detected using anti-GIGAP1 antibodies. On the other hand, GICentrin was monitored as a marker protein for membrane fractions including basal bodies. As a marker for nuclear proteins, centromeric histone $\mathrm{H} 3$ was detected in these extracts using anti-GICenH3 antibodies. Interphase cells, cytoplasmic fraction (C, lane 1), membrane fraction (M, lane 2); G1/S-phase cells: C (lane 3), M (lane 4); G2/M-phase cells: C (lane 5), M (lane 6).

\section{Acknowledgements}

We would like to thank Prof. Alexander R. Paredez (University of Washington), who provided the plasmid for transfection.

\section{Authors' contributions}

EAP, JK and SJP designed this study. EAP, JK and MYS performed the laboratory experiments. EAP, JK, and SJP analyzed and interpreted the data and wrote the manuscript. All authors read and approved the final manuscript.

\section{Funding}

This work was supported by National Research Foundation of Korea (NRF) grants funded by the Korea government (MSIT) (NRF-2020R1C1C1010581 to JK and NRF-2018R1D1A1A02085338 to SJP).

\section{Availability of data and materials}

Data supporting the conclusions of this article are included within the article and its Additional files.

\section{Declarations}

\section{Ethics approval and consent to participate}

Not applicable.

\section{Consent for publication}

Not applicable.

\section{Competing interests}

The authors declare that they have no competing interests.

Received: 21 January 2021 Accepted: 16 March 2021

Published online: 31 March 2021

\section{References}

1. Elmendorf HG, Dawson SC, McCaffery JM. The cytoskeleton of Giardia lamblia. Int J Parasitol. 2003;33:3-28.

2. Markova K, Uzlikova M, Tumova P, Jirakova K, Hagen G, Kulda J, et al. Absence of a conventional spindle mitotic checkpoint in the binucleated single-celled parasite Giardia intestinalis. Eur J Cell Biol. 2016;95:355-67.

3. Poxleitner MK, Dawson SC, Cande WZ. Cell cycle synchrony in Giardia intestinalis cultures achieved by using nocodazole and aphidicoliln. Eukayot Cell. 2008;7:569-74.

4. Reiner DS, Ankarklev J, Troell K, Palm D, Bernander R, Gillin FD, et al. Synchronisation of Giardia lamblia: identification of cell cycle stagespecific genes and a differentiation restriction point. Int J Parasitol. 2008;38:935-44.

5. Horlock-Roberts K, Reaume C, Dayer G, Ouellet C, Cook N, Yee J. Drug-free approach to study the unusual cell cycle of Giardia intestinalis. mSphere. 2017;2:e00384-16

6. Hardin WR, Li R, Xu J, Shelton AM, Alas GCM, Minin VN, et al. Myosinindependent cytokinesis in Giardia utilizes flagella to coordinate force generation and direct membrane trafficking. Proc Natl Acad Sci USA. 2017;114:E5854-63.

7. Nigg EA, Stearns T. The centrosome cycle: centriole biogenesis, duplication and inherent asymmetries. Nat Cell Biol. 2011;13:1154-60.
8. Thomas Y, Cirillo L, Panbianco C, Martino L, Tavernier N, Schwager F, et al. Cdk1 phosphorylates SPAT-1/Bora to promote PIk1 activation in C. elegans and human cells. Cell Rep. 2016;15:510-8.

9. de Cárcer G, Manning G, Malumbres M. From Plk1 to Plk5: functional evolution of polo-like kinases. Cell Cycle. 2011;10:2255-62.

10. Sunkel CE, Glover DM. Polo, a mitotic mutant of Drosophila displaying abnormal spindle poles. J Cell Sci. 1988;89:25-38.

11. Fry AM, Bayliss R, Roig J. Mitotic regulation by NEK kinase networks. Front Cell Dev Biol. 2017;5:102.

12. Bruinsma W, Raaijmakers JA, Medema RH. Switching polo-like kinase-1 on and off in time and space. Trends Biochem Sci. 2012;37:534-42.

13. Manning G, Reiner DA, Lauwaet T, Dacre M, Smith A, Zhai Y, et al. The minimal kinome of Giardia lamblia illuminates early kinase evolution and unique parasite biology. Genome Biol. 2011;12:R66.

14. Keister DB. Axenic culture of Giardia lamblia in TYI-S-33 medium supplemented with bile. Trans R Soc Trop Med Hyg. 1983;77:487-8.

15. Schindelin J, Arganda-Carreras I, Frise E, Kaynig V, Longair M, Pietzsch T, et al. Fiji: an open-source platform for biological-image analysis. Nat Methods. 2012;9:676-82.

16. Kim J, Shin MY, Park SJ. RNA-sequencing profiles of cell cycle-related genes upregulated during the G2-phase in Giardia lamblia. Korean J Parasitol. 2019;57:185-9.

17. Gourguechon S, Cande WZ. Rapid tagging and integration of genes in Giardia intestinalis. Eukaryot Cell. 2011;10:142-5.

18. Kim J, Park SJ. Roles of end-binding 1 protein and gamma-tubulin small complex in cytokinesis and flagella formation of Giardia lamblia. MicrobiologyOpen. 2019;8:e00748.

19. Carpenter ML, Cande WZ. Using morpholinos for gene knockdown in Giardia intestinalis. Eukaryot Cell. 2009;8:916-9.

20. Dawson SC, Sagolla MS, Cande WZ. The cenH3 histone variant defines centromeres in Giardia intestinalis. Chromosoma. 2007;1 16:175-84.

21. Kim J, Lee HY, Lee MA, Yong TS, Lee KH, Park SJ. Identification of a-11 giardin as a flagellar and surface component of Giardia lamblia. Exp Parasitol. 2013;135:227-33.

22. Zhang J, Chengfu Y, Wu J, Elsayed Z, Fu Z. Polo-like kinase 1-mediated phosphorylation of forkhead box protein M1b antagonizes its SUMOylation and facilitates its mitotic function. J Biol Chem. 2015;290:3708-19.

23. Colicino $E G$, Hehnly $H$. Regulating a key mitotic regulator, polo-like kinase 1 (PLK1). Cytoskeleton (Hoboken). 2018;75:481-94.

24. Kim J, Lee HY, Lee KH, Park SJ. Phosphorylation of serine 148 in Giardia Lamblia end-binding 1 protein is important for cell division. J Eukaryot Microbiol. 2017;64(4):464-80.

25. Lauwaet T, Smith AJ, Reiner DS, Romijn EP, Wong CC, Davids BJ, et al. Mining the Giardia genome and proteome for conserved and unique basal body proteins. Int J Parasitol. 2011;41:1079-92.

26. Golsteyn RM, Mundt KE, Fry AM, Nigg EA. Cell cycle regulation of the activity and subcellular localization of Plk1, a human protein kinase implicated in mitotic spindle function. J Cell Biol. 1995;129:1617-28.

27. Wakida T, Ikura M, Kuriya K, Ito S, Shiroiwa Y, Habu T, et al. The CDKPLK1 axis targets the DNA damage checkpoint sensor protein RAD9 to promote cell proliferation and tolerance to genotoxic stress. Elife. 2017;6:e29953.

28. Pintard $L$, Archambault $V$. A unified view of spatio-temporal control of mitotic entry: polo kinase as the key. Open Biol. 2018;8:180114.

29. Zitouni S, Nabais C, Jana SC, Guerrero A, Bettencourt-Dias M. Polo-like kinases: structural variations lead to multiple functions. Nat Rev Mol Cell Biol. 2014;15:433-52.

30. Bettencourt-Dias M, Rodrigues-Martins A, Carpenter L, Riparbelli M, Lehmann L, Gatt MK, et al. SAK/PLK4 is required for centriole duplication and flagella development. Curr Biol. 2005;15:2199-207.

31. Bruinsma W, Aprelia M, Kool J, Macurek L, Lindqvist A, Medema RH. Spatial separation of Plk1 phosphorylation and activity. Front Oncol. 2015;5:132.

32. Kachaner D, Garrido D, Mehsen H, Normandin K, Lavoie H, Archambault V. Coupling of polo kinase activation to nuclear localization by a bifunctional NLS is required during mitotic entry. Nat Commun. 2017:8:1701.

33. Wen D, Wu J, Wang L, Fu Z. SUMOylation promotes nuclear import and stabilization of polo-like kinase 1 to support its mitotic function. Cell Rep. 2017;21:2147-59. 
34. Li Z, Cui Q, Xu J, Cheng D, Wang X, Li B, et al. SUMOylation regulates the localization and activity of polo-like kinase 1 during cell cycle in the silkworm, Bombyx mori. Sci Rep. 2017;7:15536.

35. Gourguechon S, Holt LJ, Cande WZ. The Giardia cell cycle progresses independently of the anaphase-promoting complex. J Cell Sci. 2013;126:2246-55.

36. Kishi K, van Vugt MA, Okamoto K, Hayashi Y, Yaffe MB. Functional dynamics of polo-like kinase 1 at the centrosome. Mol Cell Biol. 2009;29:3134-50.

37. Elia AE, Rellos P, Haire LF, Chao JW, Ivins FJ, Hoepker K, et al. The molecular basis for phospho-dependent substrate targeting and regulation of Plks by the polo-box domain. Cell. 2003;115:83-95.

38. Macurek L, Lindqvist A, Lim D, Lampson MA, Klompmaker R, Freire R, et al. Polo-like kinase-1 is activated by aurora A to promote checkpoint recovery. Nature. 2008:455:119-23.

39. Tavernier N, Panbianco C, Gotta M, Pintard L. Cdk1 plays matchmaker for the polo-like kinase and its activator SPAT-1/Bora. Cell Cycle. 2015;14:2394-8.

40. Davids BJ, Williams S, Lauwaet T, Palanca T, Gillin FD. Giardia lamblia aurora kinase: a regulator of mitosis in a binucleate parasite. Int J Parasitol. 2008;38:353-69.

41. Nohynkova E, Tumova P, Kulda J. Cell division pf Giardia intestinalis: flagellar developmental cycle involves transformation and exchange of flagella between mastigonts of a diplomonad cell. Eukaryot Cell. 2006;5:753-61.
42. Dawson SC, Sagolla MS, Mancuso JJ, Woessner DJ, House SA, Fritz-Laylin $\mathrm{L}$, et al. Kinesin-13 regulates flagellar, interphase, and mitotic microtubule dynamics in Giardia intestinalis. Eukaryot Cell. 2007;6:2354-64.

43. Hoeng JC, Dawson SC, House SA, Sagolla MS, Pham JK, Mancuso JJ, et al. High-resolution crystal structure and in vivo function of a kinesin-2 homologue in Giardia intestinalis. Mol Biol Cell. 2008;19:3124-37.

44. McInally SG, Kondev J, Dawson SC. Length-dependent disassembly maintains four different flagella lengths in Giardia. Elife. 2019;8:e48694.

45. Hu Z, Liang Y, He W, Pan J. Cilia disassembly with two distinct phases of regulation. Cell Rep. 2015;10:1803-10.

46. Vasudevan KK, Jiang Y, Lechtreck KF, Kushida Y, Alford LM, Sale WS, et al. Kinesin-13 regulates the quantity and quality of tubulin inside cilia. Mol Biol Cell. 2015;26:478-94.

47. Hennessey KM, Alas GCM, Rogier I, Li R, Merritt EA, Paredez AR. Nek8445, a protein kinase required for microtubule regulation and cytokinesis in Giardia lamblia. Mol Biol Cell. 2020;31:1611-22.

\section{Publisher's Note}

Springer Nature remains neutral with regard to jurisdictional claims in published maps and institutional affiliations.
Ready to submit your research? Choose BMC and benefit from:

- fast, convenient online submission

- thorough peer review by experienced researchers in your field

- rapid publication on acceptance

- support for research data, including large and complex data types

- gold Open Access which fosters wider collaboration and increased citations

- maximum visibility for your research: over $100 \mathrm{M}$ website views per year

At BMC, research is always in progress.

Learn more biomedcentral.com/submissions 